\title{
Neutrophil pyroptosis mediates pathology of $P$. aeruginosa lung infection in the absence of the NADPH oxidase NOX2
}

\author{
J-C Ryu ${ }^{1,2}$, M-J Kim ${ }^{1,2}$, Y Kwon ${ }^{1,2}$, J-H Oh ${ }^{1,2}$, SS Yoon ${ }^{2,3}$, SJ Shin ${ }^{2,3}$, J-H Yoon ${ }^{1,2,4,5}$ and J-H Ryu ${ }^{2,6}$
}

Nod-like receptor family, CARD domain-containing 4 (NLRC4) inflammasome activation is required for efficient clearance of intracellular pathogens through caspsase-1-dependent pyroptosis in macrophages. Although neutrophils have a critical role in protection from Pseudomonas aeruginosa infection, the mechanisms regulating inflammasomemediated pyroptosis in neutrophils and its physiological role are largely unknown. We sought to determine the specific mechanisms regulating neutrophil pyroptosis in P. aeruginosa strain PAO1 (PAO1) lung infection and to identify the pathological role of this process. Nox2 $2^{-1-}$ models with reduced neutrophil antibacterial activity exhibited increased neutrophil pyroptosis, which was mediated by flagellin, a pathogenic PAO1 component. We also demonstrate that PAO1-induced pyroptosis depended on NLRC4 and Toll-like receptor 5 (TLR5) in neutrophils generated from NIrc4 ${ }^{-1-}$ or $\mathrm{TIr} 5^{-1-}$ mice. Our study reveals previously unknown mechanisms and physiological role of neutrophil pyroptosis during $P$. aeruginosa lung infection. Furthermore, our findings regarding neutrophil pyroptosis in the context of neutrophil dysfunction may explain the causes of acute and/or chronic infectious diseases discovered in immunecompromised patients.

\section{INTRODUCTION}

Pseudomonas aeruginosa is a major pathogen responsible for nosocomial pneumonia and is associated with high morbidity and mortality rates. ${ }^{1-3}$ As $P$. aeruginosa infections are most frequently seen in individuals with compromised immune systems, the host immune response is clearly essential in both preventing and controlling infection. ${ }^{4,5}$ The bacterial protein flagellin is translocated to the cytosol, where it is efficiently recognized by NLRC4 (Nod-like receptor family, CARD domain-containing 4) ${ }^{6-10}$ Several lines of evidence support an essential role for the NLRC4-coupled inflammasome in caspase-1 (CASP1) activation in response to Shigella flexneri, ${ }^{6}$ Salmonella typhimurium, ${ }^{11}$ and Legionella pneumophila. ${ }^{12}$ In these reports, mouse models infected with these pathogens showed flagellin-induced activation of NLRC4 inflammasome/ CASP1, leading to the secretion of proinflammatory cytokines such as interleukin-1 $\beta$ (IL-1 $\beta$ ) and IL-18 and induction of a rapid lytic form of inflammatory cell death known as pyroptosis. For host protection from pathogen invasion, IL-1 $\beta$ and IL-18 induce phagocyte recruitment and activation. ${ }^{13-15}$ In turn, pyroptosis releases the intracellular pathogens into the extracellular environment, exposing them to neutrophil-mediated destruction. ${ }^{16-19}$ However, although these NLRC4/CASP1-dependent responses aid clearance of intracellular pathogens, NLRC4 activation by $P$. aeruginosa decreases the clearance of this pathogen and increases disease pathology in acute lung infection. ${ }^{20,21}$ In acute lung infection, neutrophils, rather than macrophages, are the first cell type recruited in large quantities to the site of infection and are thus involved in the initial clearance of infected cells. Genetic disorders leading to abnormal neutrophil function characterize up to $20 \%$ of all reported primary immune deficiencies. ${ }^{22}$

${ }^{1}$ Research Center for Natural Human Defense System, Yonsei University College of Medicine, Seoul, Korea. ${ }^{2}$ Brain Korea 21 PLUS Project for Medical Science, Yonsei University College of Medicine, Seoul, Korea. ${ }^{3}$ Department of Microbiology and Institute for Immunology and Immunological Diseases, Yonsei University College of Medicine, Seoul, Korea. ${ }^{4}$ Department of Otorhinolaryngology, Yonsei University College of Medicine, Seoul, Korea. ${ }^{5}$ The Airway Mucus Institute, Yonsei University College of Medicine, Seoul, Korea and ${ }^{6}$ Severance Biomedical Science Institute, Yonsei University College of Medicine, Seoul, Korea. Correspondence: J-H Ryu (yjh@yuhs.ac) 
Although neutrophils have been shown to have a critical role in the primary protection from pathogen infection, most studies of the NLRC4 inflammasome have focused on its regulatory role in the context of macrophages. However, NLRC4 was recently reported to be expressed in neutrophils and to be responsible for CASP1-dependent IL-1 $\beta$ secretion during challenge with Salmonella or $P$. aeruginosa..$^{11,23-25}$ In contrast to inflammasome-dependent IL-1 $\beta$ secretion in pathogen-infected neutrophils, the regulatory mechanisms of neutrophil pyroptosis upon phagocytosis of pathogens are not well defined. This primarily stems from the assumption that neutrophils are not likely to undergo pyroptosis, because their antibacterial activity is so strong that pathogens do not have enough time to proliferate and trigger pyroptosis. To test this assumption, we developed a NADPH oxidase 2 (NOX2)-deficient condition in which hypochlorous acid (HOCl), a reactive oxygen species (ROS) with powerful antibacterial activity, would not be generated. ${ }^{26,27}$ In wild-type (WT) mice $\left(N o x 2^{+/+}\right)$, the percentages of pyroptotic neutrophils were extremely low at $24 \mathrm{~h}$ after $P$. aeruginosa infection (about $0.7 \%$ ); meanwhile, under the same condition, neutrophils from Nox $2^{-/-}$mice showed marked increases in pyroptotic cell death, a $>15$-fold increase (approximately 11\%). Thus we hypothesized that neutrophil pyroptosis might be induced by acute $P$. aeruginosa infection via inflammasome signaling in conditions of lower neutrophil antimicrobial activity in which bacterial burden is increased. In this study, we demonstrate that flagellin within $P$. aeruginosa mediates neutrophil pyroptosis upon acute lung infection through CASP1-dependent signaling in Nox $2^{-/}$mice, leading to greater inflammation and lung injury as a result of increased $P$. aeruginosa burden in the lungs. Our study outlines mechanisms that were previously unknown and the pathological role of neutrophil pyroptosis during $P$. aeruginosa lung infection upon neutrophil dysfunction.

\section{RESULTS \\ Nox2 ${ }^{-l-}$ mice show increased bacterial burden, lung injury, and mortality after challenge with PAO1}

To know the physiological role of NOX2 in acute lung infection, we first determined 4-day survival rates in a lung infectious mouse model by pulmonary challenge with PAO1 $\left(4 \times 10^{5}\right.$ colonyforming units (CFU)) using NOX2-deficient mice (Nox2 ${ }^{-1-}$ ). Notably, Nox2 $2^{-1-}$ mice were more susceptible to PAO1 infection than their WT littermates $\left(\right.$ Nox $\left.2^{+/+}\right)$. At 3-4 days after PAO1 challenge, nearly all of the Nox $2^{-1-}$ mice had died, whereas all of the Nox $2^{+/+}$mice survived beyond 3 days (Figure 1a). In accordance with their increased mortality, Nox $2^{-1-}$ mice had decreased pulmonary clearance of bacteria and significantly higher bacterial burdens in their bronchoalveolar lavage (BAL) fluid (Figure 1b). To determine whether the increased bacterial burden of Nox2 $2^{-1-}$ mice was accompanied by increased lung damage, we quantified the levels of protein and immunoglobulin $\mathrm{M}$ (IgM) in BAL fluid. Nox $2^{-1-}$ mice exhibited more lung injury than Nox $2^{+/+}$mice at both 12 and $24 \mathrm{~h}$ after PAO1 infection (Figure 1c,d). Fibrin deposition in the alveolar spaces of Nox $2^{-I-}$ mice was also much higher than in Nox2 $2^{-I-}$ mice
(Figure 1e,f). Elevated levels of various inflammatory cytokines, including tumor necrosis factor- $\alpha$ (TNF- $\alpha$ ) (Figure 1g), IL-1 $\beta$ (Figure 1h), and IL-6 (Figure 1i), were also observed in Nox $2^{-1-}$ mice. To determine whether the increased bacterial burden and extensive lung injury in the lungs of Nox $2^{-1-}$ mice during PAO1 infection leads to severe systemic infection, we evaluated the CFUs in the liver and spleen and also assessed organ dysfunction by measuring serum biochemical indicators. The CFUs in the liver and spleen of Nox2 $2^{-1}$ mice were significantly increased compared with those in Nox2 $2^{+/+}$mice; similar results were obtained for the blood urea nitrogen, aspartate transaminase, creatinine, and alanine transaminase levels (Supplementary Figure S1a-f). These results indicate that NOX2 is critical for host protection against acute lung infection with PAO1 through clearance of bacteria.

\section{Neutrophils, rather than macrophages, are essential for host protection during PAO1 lung infection}

We next examined the numbers of intact neutrophils and macrophages in the bronchoalveolar spaces of Nox2 $2^{+/+}$and Nox $2^{-1-}$ mice during PAO1 infection. In Nox $2^{+/+}$mice, the number of neutrophils was significantly increased at $12 \mathrm{~h}$ and decreased at $24 \mathrm{~h}$, while the number of macrophages was only slightly increased at 12 and $24 \mathrm{~h}$ (Figure 2a). Interestingly, the number of neutrophils was dramatically decreased in Nox2 $2^{-1-}$ mice compared with that in Nox2 $2^{+/+}$mice (Figure 2a). To determine whether neutrophils or macrophages are essential for bacteria clearance and host lung protection against PAO1 infection, we counted the CFUs and assessed the extent of lung injury in PAO1 infection after depleting neutrophils or macrophages, respectively. Administration of $\alpha$-Ly6G antibody and clodronate liposomes efficiently depleted neutrophils (Figure 2b) and macrophages (Figure 2c), respectively, from the BAL fluid of WT mice. Much higher CFU counts (Figure 2d), levels of BAL protein (Figure 2e), and levels of IgM (Figure 2f) were detected in neutrophil-depleted mice compared with control mice, while none of these parameters were affected in macrophage-depleted mice (Figure 2d-f). Much higher levels of proinflammatory cytokines such as TNF- $\alpha$, IL- $1 \beta$, and IL-18 were observed in neutrophil-depleted mice, while cytokine levels were not affected in macrophage-depleted mice (Figure 2g-i). Because bacteria-mediated activation of the production of $\mathrm{HOCl}^{-}$, a compound with antibacterial activity in neutrophils, depends on NOX2, ${ }^{26-28}$ we investigated whether NOX2 exhibits the capability of directly killing PAO1 ingested by neutrophils. To do so, we infected PAO1 into bone marrow-derived neutrophils (BMDNs) extracted from Nox2 $2^{+/+}$and Nox2 $2^{-1-}$ mice for $30 \mathrm{~min}$, followed by treatment with gentamicin to clear extracellular PAO1. We then examined the viability of intracellular PAO1 within Nox $2^{+/+}$or Nox $2^{-1-}$ BMDNs. Nox $2^{-1-}$ BMDNs showed higher number of viable PAO1, compared with Nox2 $2^{+/+}$BMDNs (Supplementary Figure $\mathbf{S 2 a}, \mathbf{b})$, indicating that NOX2 is required for direct killing of neutrophil-ingested PAO1. Through fluorescence-activated cell sorting (FACS) analysis capable of probing specifically 
a

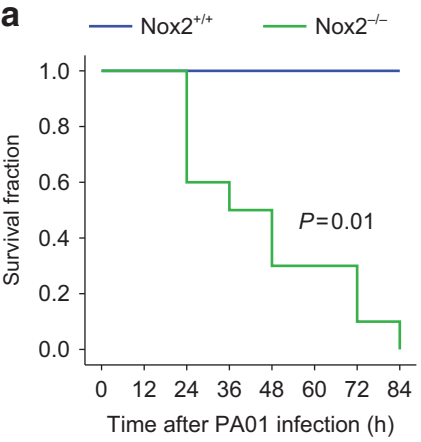

b

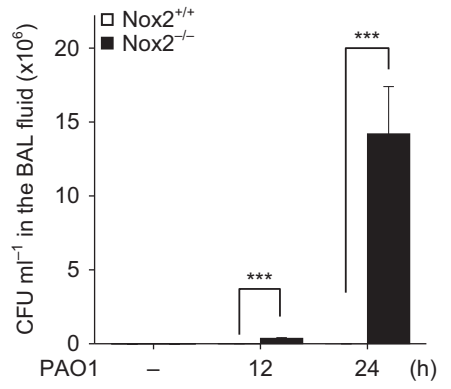

C

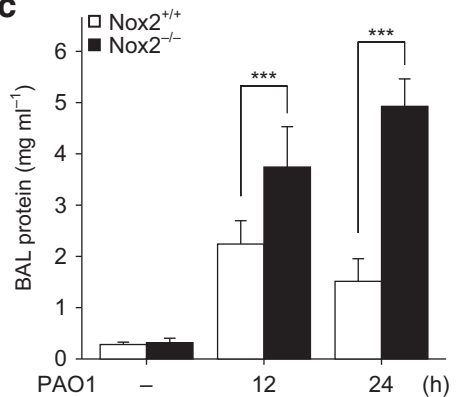

\section{d}

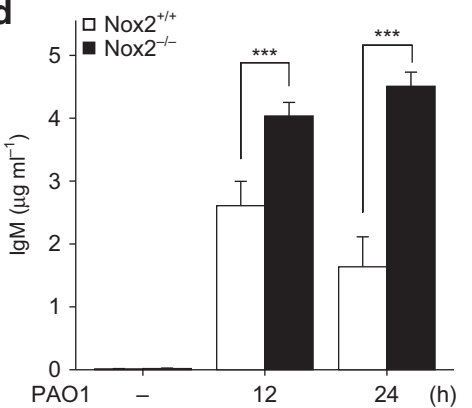

e

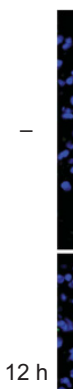

$\mathrm{NOX}^{+++}$

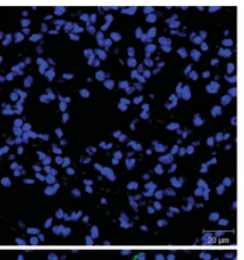

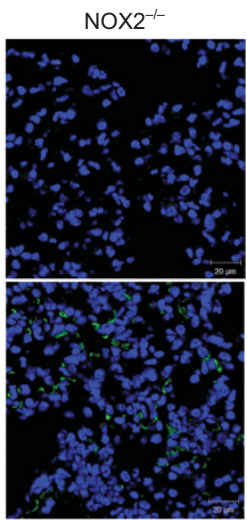

f
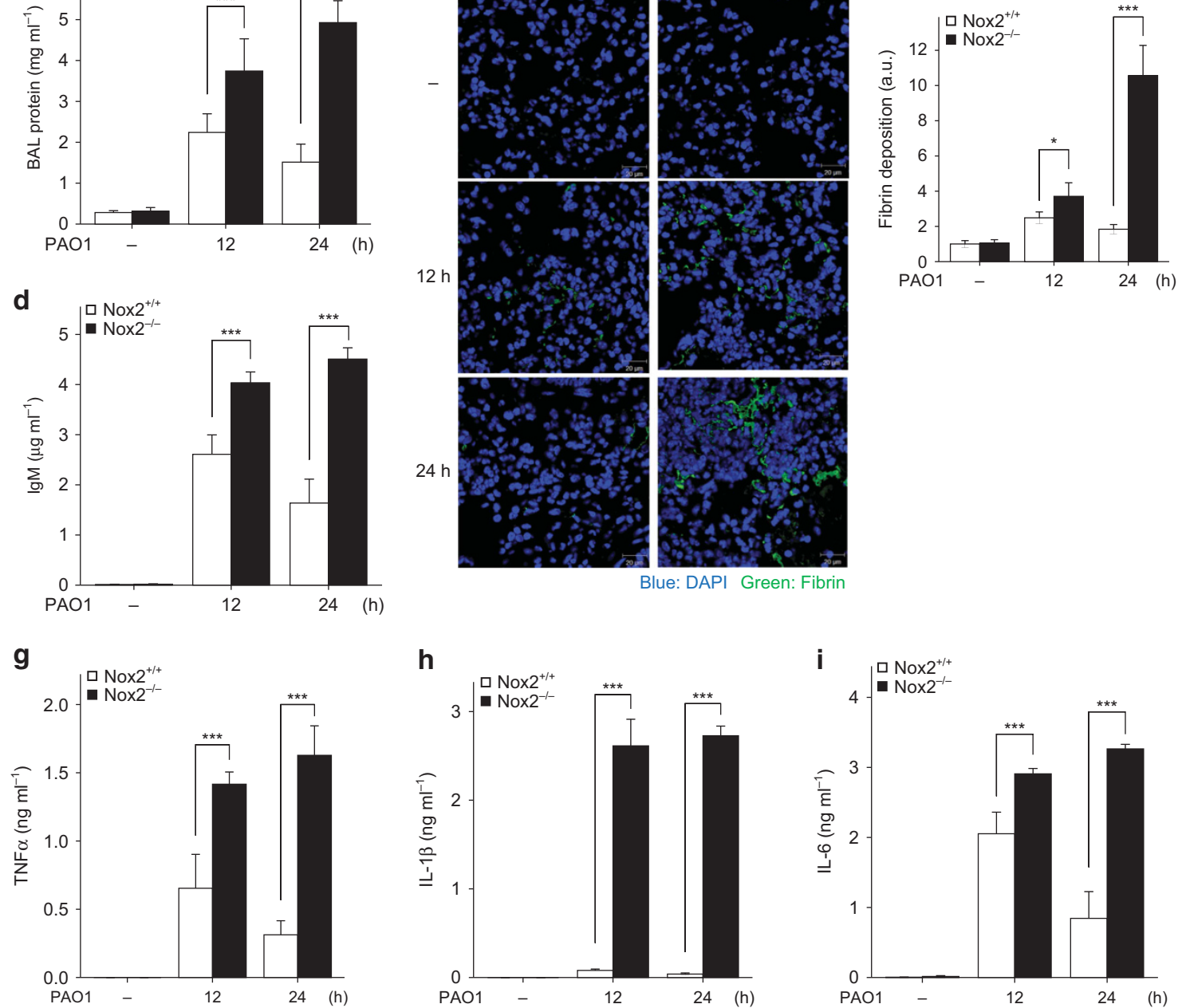

h

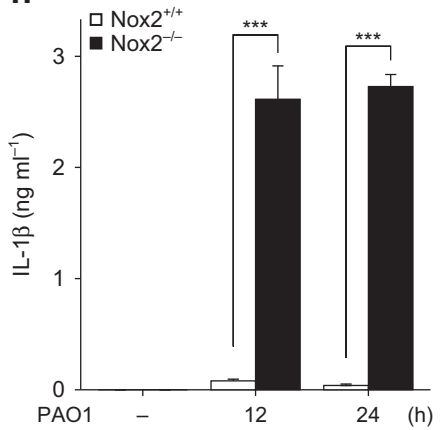

i $\quad \mathrm{Nox}^{+/+}$

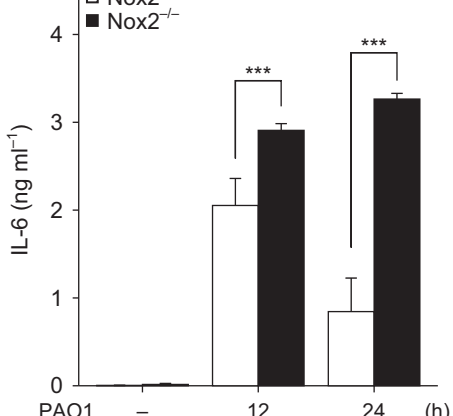

Figure 1 Nox2 $2^{-1-}$ mice show increased bacterial burden and lung injury upon PAO1 infection, contributing to their increased mortality. (a-i) Mice were instilled intranasally with $4 \times 10^{5}$ colony-forming units (CFU) of PAO1. (a) The survival rates of Nox2 $2^{-1-}$ and Nox2 $2^{+1+}$ mice were monitored for 4 days $(n=10)$. (b) Nox2 $2^{-/-}$and $\mathrm{Nox}^{+/+}$mice were killed at 12 and $24 \mathrm{~h}$ postinfection and the bacterial burdens in the bronchoalveolar lavage (BAL) fluid were

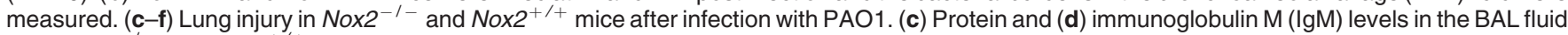
from Nox2 $2^{-1-}$ and Nox2 $2^{+/+}$mice were determined at 12 and $24 \mathrm{~h}$ after intranasal instillation of PAO1. (e and f) Fibrin deposits in lung tissue sections of $\mathrm{Nox}^{-1-}$ and $\mathrm{Nox}^{+/+}$mice at 12 and $24 \mathrm{~h}$ after intranasal instillation of PAO1. (g-i) Cytokine levels in BAL fluid after infection with PAO1. Levels of (g)

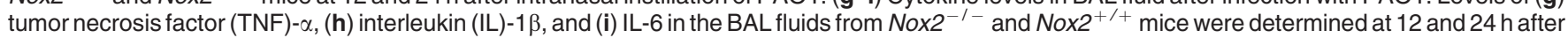
intranasal instillation of PAO1. ${ }^{\star} P<0.05 ;{ }^{* \star} P<0.001$.

for $\mathrm{HOCl}$, we also demonstrated increases in $\mathrm{HOCl}^{-}$in Nox $2^{+/+}$neutrophils upon PAO1 lung infection, whereas this effect was not observed in Nox2 $2^{-/-}$neutrophils
(Supplementary Figure S3). These results indicate that NOX2 is critical for the maintenance of intact neutrophils via clearance of neutrophil-ingested PAO1. 


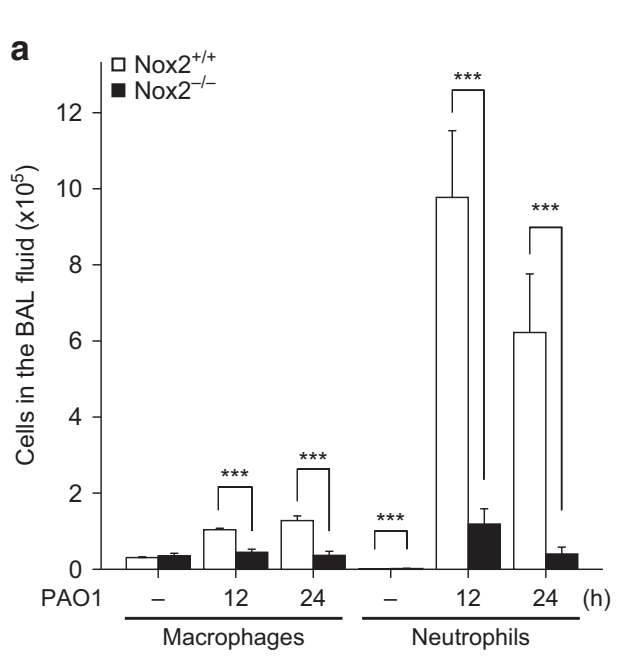

d

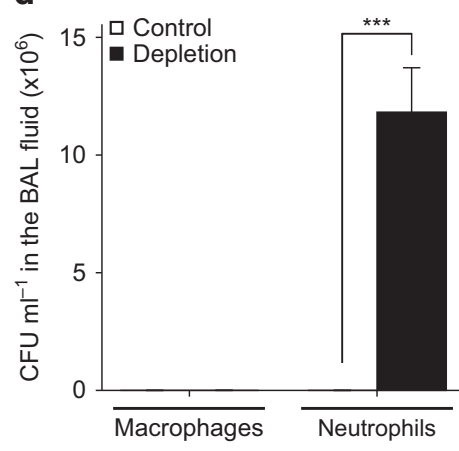

g

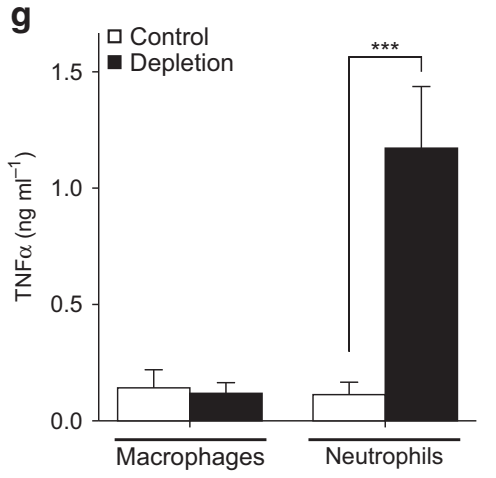

b

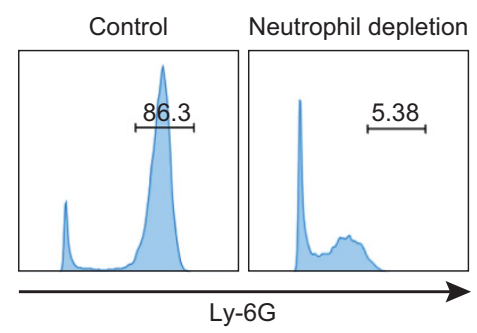

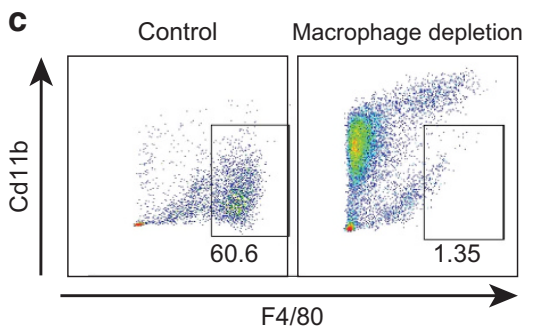

f e

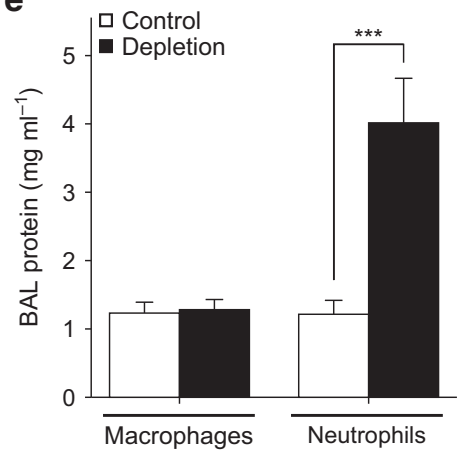

h

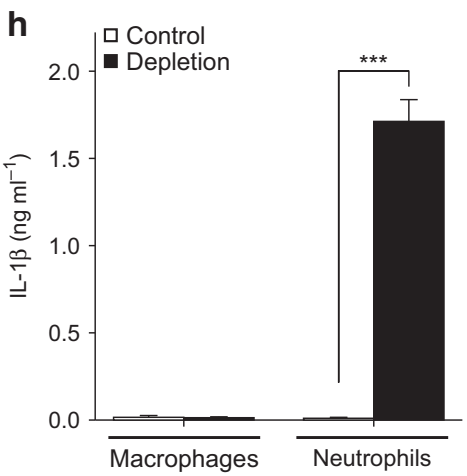

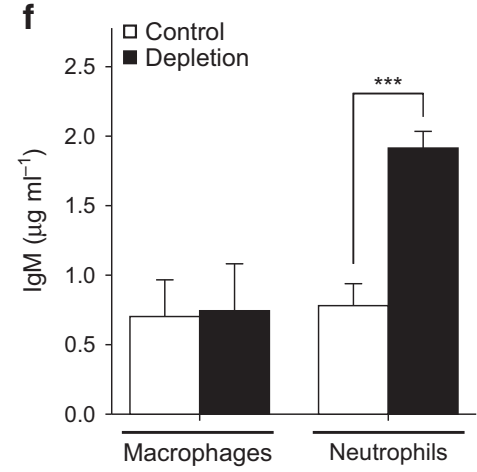

i

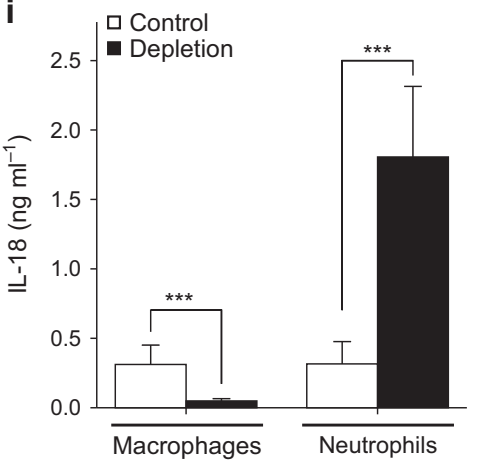

Figure 2 Nox2 $2^{-1-}$ mice show fewer neutrophils in the bronchoalveolar space during PAO1 lung infection. (a) The numbers of macrophages and neutrophils in the bronchoalveolar lavage (BAL) fluid were determined at 12 and $24 \mathrm{~h}$ after intranasal instillation of $4 \times 10^{5}$ colony-forming units (CFU) of PAO1. (b) The extent of neutrophil depletion from BAL fluid was determined $8 \mathrm{~h}$ after PAO1 infection by flow cytometry. (c) The extent of macrophage depletion from BAL fluid was determined without PAO1 infection by flow cytometry. (d-i) Bacterial burdens, lung injury, and cytokine levels in the BAL fluid from neutrophil-depleted mice after PAO1 infection. (d) Bacterial burdens, (e) BAL protein, (f) immunoglobulin M (IgM), (g) tumor necrosis factor (TNF)- $\alpha$,

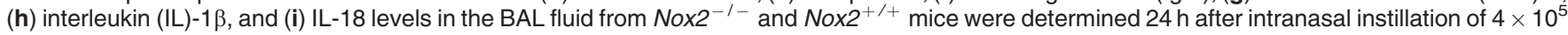
CFU of PAO1. ${ }^{* *} P<0.001$.

\section{Neutrophil pyroptosis is increased in Nox2 $2^{-1-}$ mice during acute lung infection}

As fewer intact neutrophils and higher PAO1 burdens were observed in the bronchoalveolar space of $N o x 2^{-1-}$ mice compared with that of $\mathrm{Nox}^{+/+}$mice during acute infection, we postulated that more neutrophils in Nox $2^{-1-}$ mice may undergo cell death than in Nox2 $2^{+/+}$mice. We first measured neutrophil cell death within the bronchoalveolar space through FACS analysis using propidium iodide (PI) or Annexin V

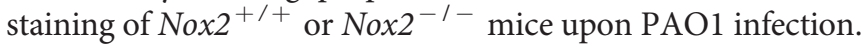
The percentage of necrotic cells $\left(\mathrm{PI}^{+} /\right.$Annexin $\left.\mathrm{V}^{+}\right)$was dramatically increased in Nox2 ${ }^{-1-}$ neutrophils compared with 

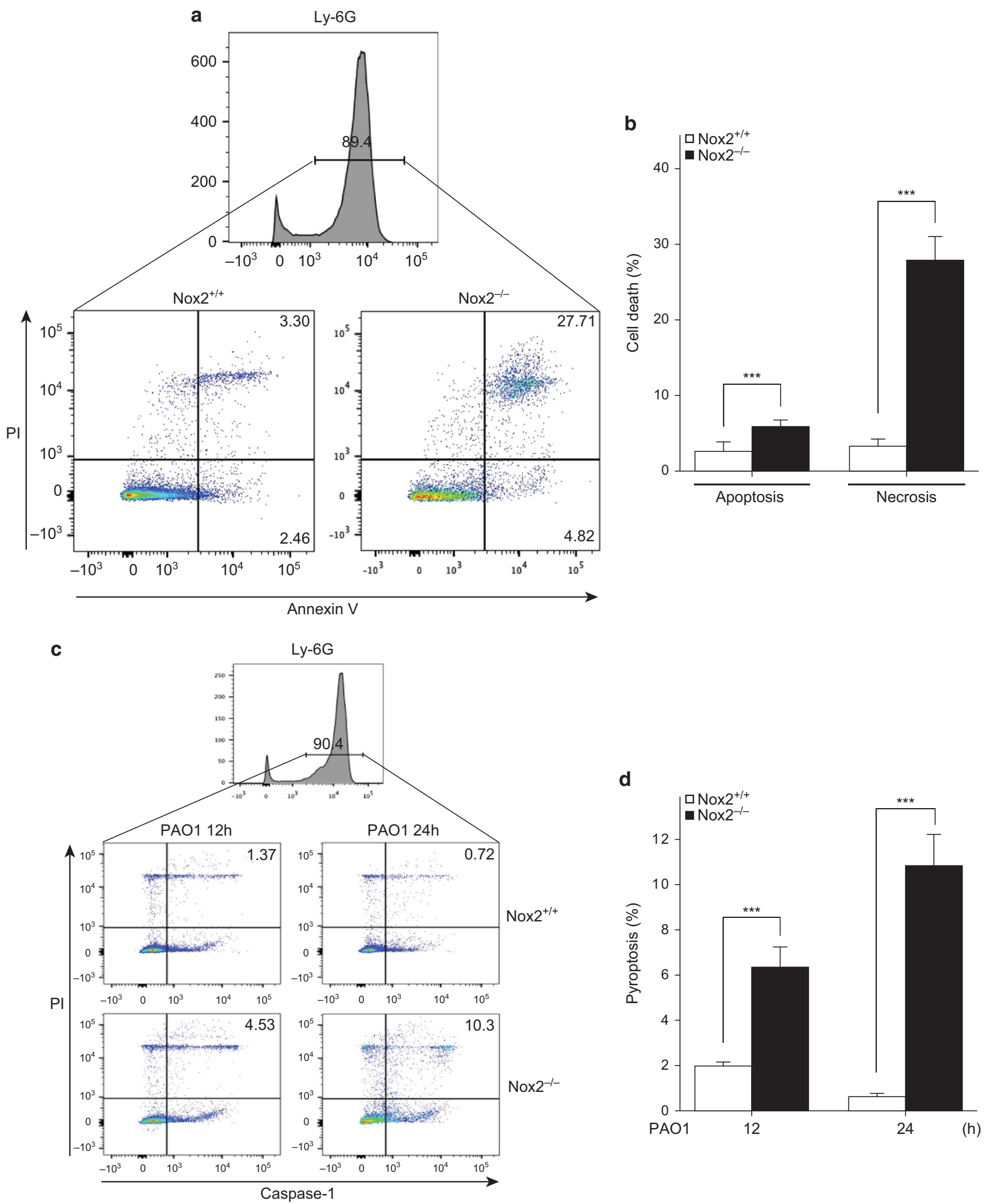

Figure 3 Neutrophil pyroptosis is increased in the lungs of Nox2 $2^{-1-}$ mice during PAO1 infection. (a and $\left.\mathbf{b}\right)$ Neutrophil cell death in the bronchoalveolar lavage (BAL) fluid from PAO1-infected mice. (a) Representative flow cytometric plots and (b) the percentages of neutrophil cell death in the BAL fluids from Nox2 $2^{-1-}$ and $\mathrm{Nox}^{+/+}$mice were determined $24 \mathrm{~h}$ after intranasal instillation of $4 \times 10^{5}$ colony-forming units (CFU) of PAO1. (c and d) Neutrophil pyroptosis in the BAL fluid from PAO1-infected mice. (c) Representative flow cytometric plots and (d) the percentages of neutrophil pyroptosis in the BAL fluids from Nox2 $2^{-1-}$ and $\mathrm{Nox}^{+/+}$mice were determined at 12 and $24 \mathrm{~h}$ after intranasal instillation of $4 \times 10^{5} \mathrm{CFU}$ of PAO1. ${ }^{* \star *} \mathrm{P}<0.001$. 
Nox $2^{+/+}$neutrophils upon infection, whereas the percentage of apoptotic cells $\left(\mathrm{PI}^{-} /\right.$Annexin $\mathrm{V}^{+}$) was slightly increased in

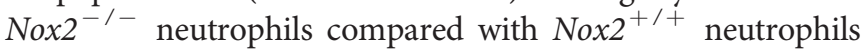
(Figure 3a,b). We next hypothesized that pyroptosis, a lytic form of programmed cell death, is induced in neutrophils in PAO1-infected Nox2 $2^{-1-}$ mice. A basic event in pyroptosis is the early loss of plasma membrane integrity by pathogen infection, and thus the lactate dehydrogenase (LDH) release assay has primarily been used to characterize cell death by pyroptosis in macrophages. ${ }^{7,29,30}$ Thus we measured LDH release, as well as the numbers of $7 \mathrm{AAD}^{+}$cells or both active $\mathrm{CASP}^{+}{ }^{+}$and $\mathrm{PI}^{+}$cells, using FACS analysis in PAO1-infected bone marrow-derived macrophages (BMDMs) extracted from WT mice. Both $\mathrm{LDH}$ release and the number of $7 \mathrm{AAD}^{+}$cells or both active CASP1 ${ }^{+}$and $\mathrm{PI}^{+}$cells were increased by PAO1 infection in similar patterns (Supplementary Figure S4a-e). We also showed that active CASP1 and IL-1 $\beta$ secretion are strongly expressed in BMDMs upon PAO1 infection using western blotting analysis (Supplementary Figure S4f). We compared the levels of neutrophil pyroptosis in $\mathrm{Nox}^{+/+}$vs. Nox $2^{-1-}$ mice upon PAO1 infection using a FACS strategy to detect both active CASP $1^{+}$and $\mathrm{PI}^{+}$cells. In Nox2 ${ }^{+/+}$mice, the percentage of pyroptotic neutrophils was extremely low at $24 \mathrm{~h}$ after PAO1 infection (about 0.7\%), while neutrophils from Nox $2^{-1-}$ mice showed marked pyroptotic cell death, with more than a 15-fold increase (approximately 11\%) in the same condition (Figure 3c,d). A higher number of neutrophils showing the necrotic phenotype, including loss of cell membrane integrity and release of cellular contents, and an increased load of extracellular bacteria were observed in the bronchoalveolar space of infected Nox2 $2^{-1-}$ mice compared with $\mathrm{Nox}^{+/+}$mice (Supplementary Figure S5a,b). These results indicate that NOX2 suppresses neutrophil pyroptosis during PAO1 lung infection.

\section{Neutrophils derived from Nox2 ${ }^{-1-}$ mice show higher bacterial burdens and more pyroptosis upon PAO1 infection}

We next investigated whether the increase in neutrophil pyroptosis in Nox2 $2^{-1-}$ mice depends on the amount of neutrophil-internalized bacteria. We first infected BMDNs extracted from $\mathrm{Nox}_{2}{ }^{+/+}$or $\mathrm{Nox} 2^{-1-}$ mice with green fluorescent protein (GFP)-tagged PAO1 (PAO1-GFP) to determine the efficiency of PAO1-GFP infection. The number of Nox2 $2^{-1-}$ BMDNs containing PAO1-GFP was not significantly different than that of Nox2 $2^{+/+}$BMDNs with PAO1GFP at $10 \mathrm{~min}$ postinfection (Figure $4 \mathbf{a}, \mathbf{b}$ ). However, The number of Nox2 $2^{-1-}$ BMDNs containing PAO1-GFP were more increased than that of Nox2 $2^{+1+}$ BMDNs containing PAO1-GFP at $60 \mathrm{~min}$ postinfection (Figure 4a,b), indicating that NOX2 is responsible for clearance of neutrophil-ingested PAO1 but not for initial PAO1 uptake. In accordance with the rates of PAO1 uptake, pyroptosis was significantly increased in Nox $2^{-1-}$ BMDNs with PAO1-GFP at $60 \mathrm{~min}$, but not at $10 \mathrm{~min}$, postinfection (Figure $\mathbf{4 c}, \mathbf{d}$ ). Western blotting analysis confirmed that CASP1 is more efficiently processed in
Nox $2^{-1-}$ BMDNs than in Nox $2^{+/+}$BMDNs, as evidence by the increased levels of its mature form (CASP1 p20) at 30 and $60 \mathrm{~min}$ after PAO1 infection (Figure 4e). Furthermore, Nox $2^{-1-}$ BMDNs had secreted more IL- $1 \beta$ than Nox $2^{+/+}$ BMDNs at $60 \mathrm{~min}$ after PAO1 infection (Figure 4f). However, secretion of IL-18, another CASP1-dependent cytokine, was

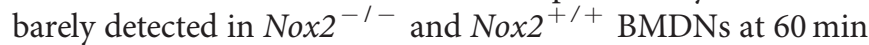
after PAO1 infection (data not shown). These results indicate that the increased bacterial burden in Nox2 $2^{-1-}$ BMDNs contributes to the increased pyroptosis.

\section{PAO1 infection-induced mitochondrial ROS is responsible for neutrophil pyroptosis}

To examine the effect of PAO1 infection on mitochondrial integrity in neutrophils, we evaluated the functional mitochondrial pool in PAO1-infected BMDNs. To this end, we used tetramethylrhodamine ethyl ester (TMRE), a fluorescent probe sensitive to mitochondrial membrane potential, to assess mitochondrial depolarization. More mitochondrial depolar-

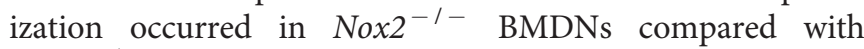
Nox2 $2^{+/+}$BMDNs upon PAO1 infection, while it was not affected in the absence of PAO1 infection (Figure 5a,b). In accordance with these TMRE results, the level of mitochondrial ROS in Nox2 $2^{-1-}$ BMDNs was much higher than that in Nox $2^{+/+}$BMDNs upon PAO1 infection (Figure $\mathbf{5 c}, \mathbf{d}$ ).

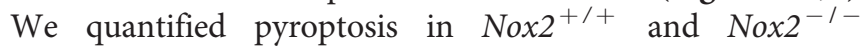
BMDNs upon PAO1 infection after treating cells with Mito-Tempo, a mitochondrial ROS scavenging reagent. The PAO1-mediated increase in pyroptosis in Nox2 $2^{-1-}$ BMDNs was significantly decreased in the presence of Mito-Tempo (Figure 5e). The increased levels of CASP1 activation and IL-1 $\beta$ in Nox $2^{-1-}$ BMDNs were also blunted in the presence of MitoTempo (Figure 5f,g).

\section{PAO1-infected neutrophils are responsible for both cell death and IL-1 $\beta$ secretion through CASP1-dependent signaling in NOX2-deficient condition}

We examined cell death and IL-1 $\beta$ secretion in Nox2 $2^{+/+}$ BMDNs and Nox2 $2^{-1-}$ BMDNs upon PAO1 infection in the presence of Ac-YVAD-CHO, a CASP1 inhibitor. Therein, increases in cell death (Figure 6a-c) and IL-1 $\beta$ secretion (Figure 6d,e) in PAO1-infected Nox2 $2^{-1-}$ BMDNs were reduced dramatically upon Ac-YVAD-CHO treatment. To verify the pathological effect of CASP1 under a NOX2-deficient condition, we evaluated neutrophil cell death, PAO1 burden in the lungs, lung injuries, and proinflammatory cytokines in Nox $2^{+/+}$and Nox $2^{-1-}$ mice upon acute lung infection in the presence of Ac-YVAD-CHO. In accordance with results in BMDNs, increases in neutrophil cell death (Figure 7a,b), PAO1 burden (Figure 7c), lung injuries (Figure $7 \mathbf{d}, \mathbf{e}$ ), and proinflammatory cytokines (Figure $\mathbf{7} \mathbf{f}-\mathbf{h}$ ) were significantly reduced in PAO1-infected Nox2 $2^{-1-}$ mice treated with AcYVAD-CHO. These results demonstrate that PAO1-infected neutrophils are responsible for both IL- $1 \beta$ secretion and cell death through CASP1-dependent signaling in NOX2-deficient neutrophils in in vitro and in vivo conditions. 
a

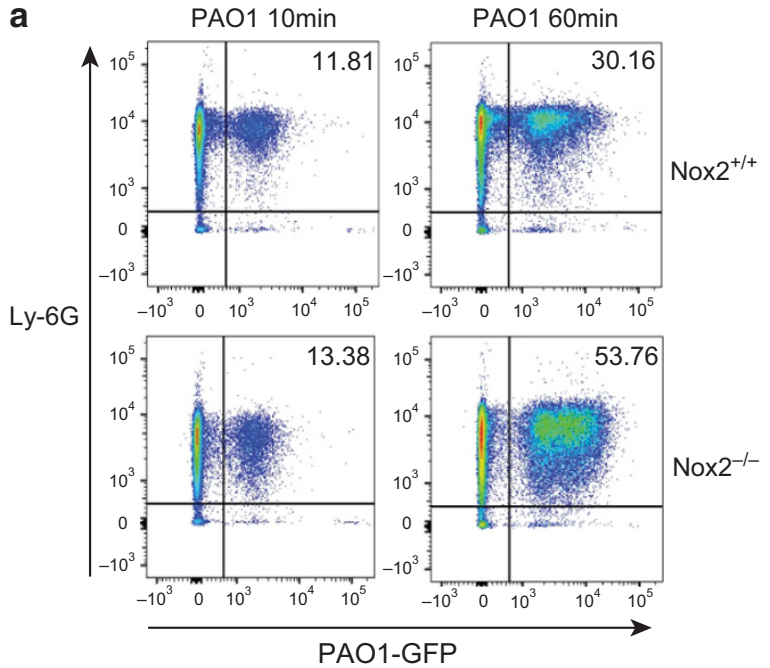

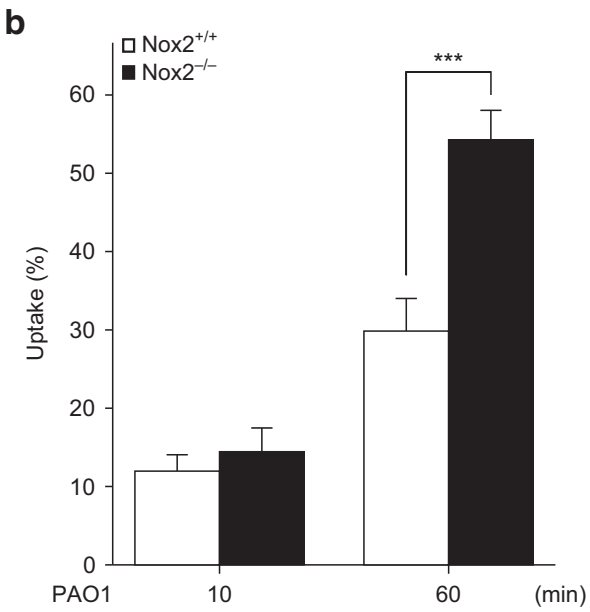

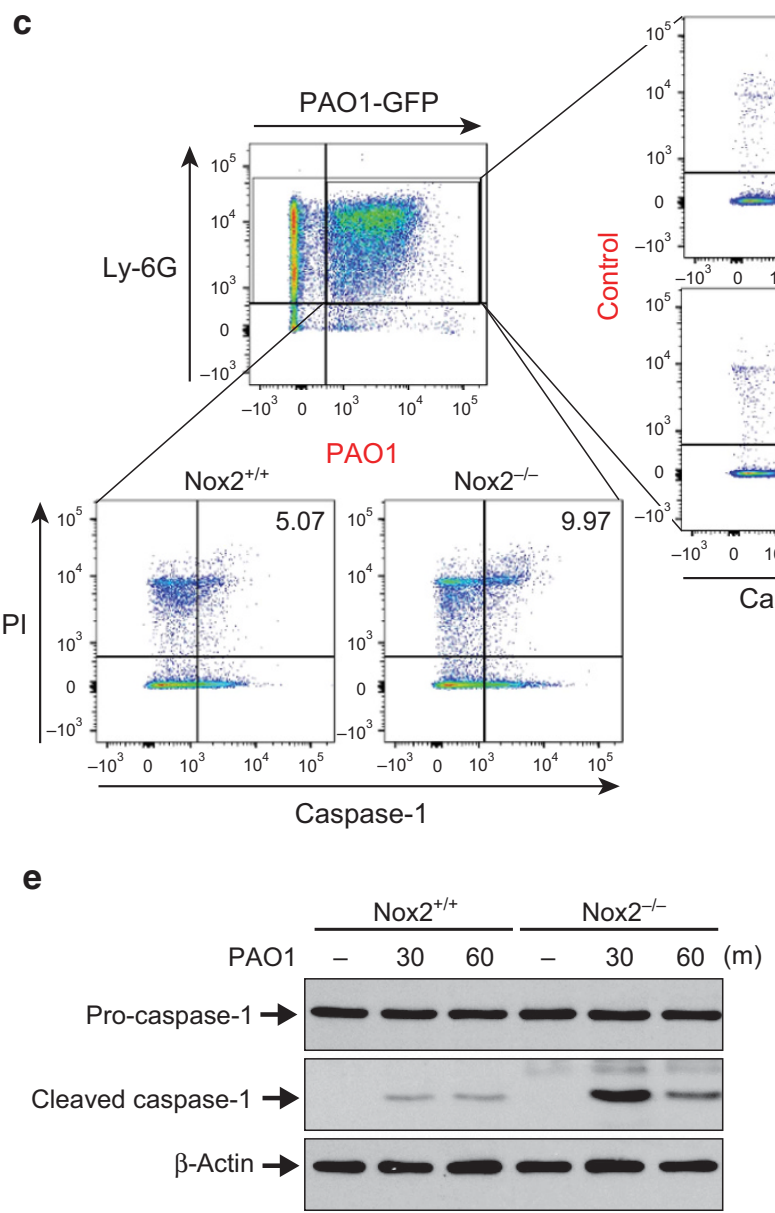

d

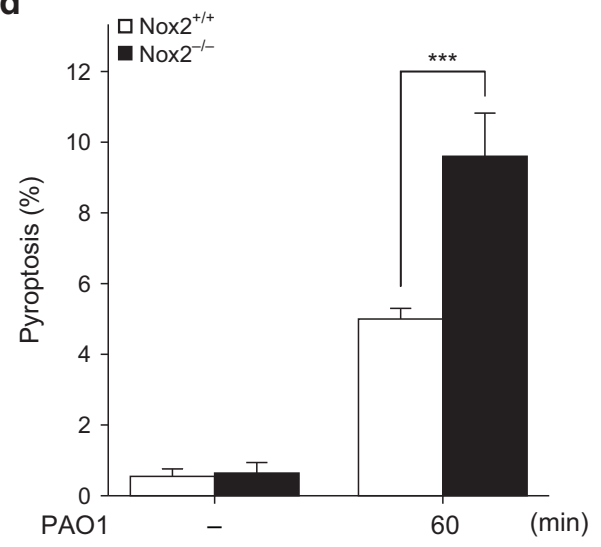

$\mathbf{f}$

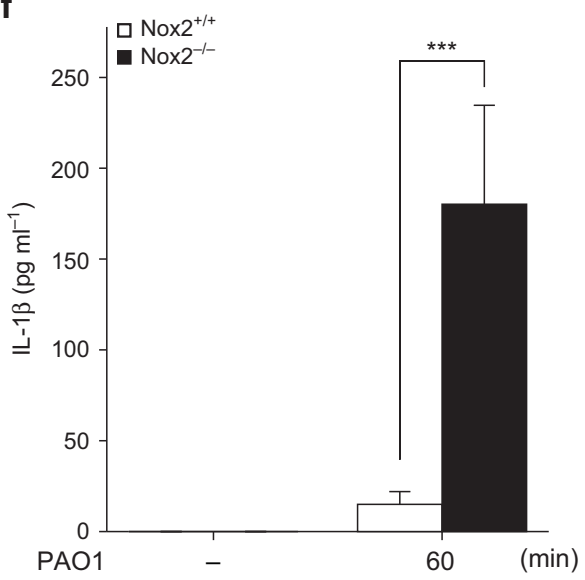

Figure 4 Neutrophils derived from Nox2 ${ }^{-1-}$ mice show higher bacterial burdens and increased pyroptosis upon PAO1 infection. (a-f) Bone marrowderived neutrophils (BMDNs) extracted from Nox2 ${ }^{-1-}$ mice and Nox2 $2^{+/+}$mice were infected with green fluorescent protein (GFP)-tagged PAO1

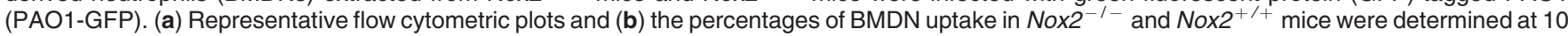
and 60 min after infection with PAO1-GFP (multiplicity of infection (MOI) 20). (c) Representative flow cytometric plots and (d) the percentages of pyroptotic BMDNs, including PAO1-GFP, in Nox2 ${ }^{-1-}$ and Nox2 ${ }^{+/+}$mice were determined $1 \mathrm{~h}$ after infection with PAO1-GFP (MOI 20). (e and f) BMDNs were infected with PAO1 (MOI 20). Cell extracts and their supernatants were harvested at 30 and 60 min postinfection. (e) Expression of cleaved CASP1 in cell extracts was detected by western blotting. (f) The levels of interleukin (IL)-1 $\beta$ in cell supernatants were detected by enzyme-linked immunosorbent assay. ${ }^{* \star *} P<0.001$. 
a

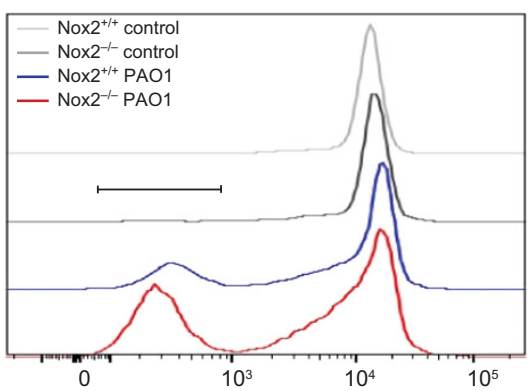

C

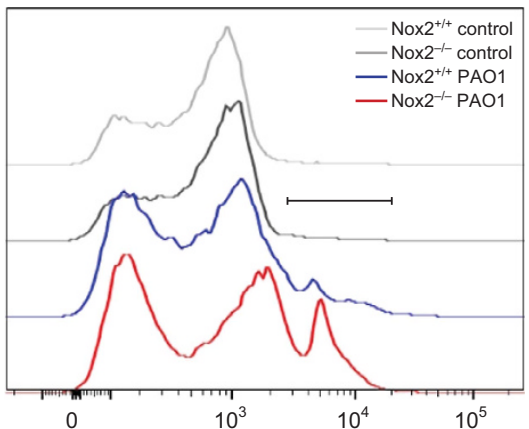

e

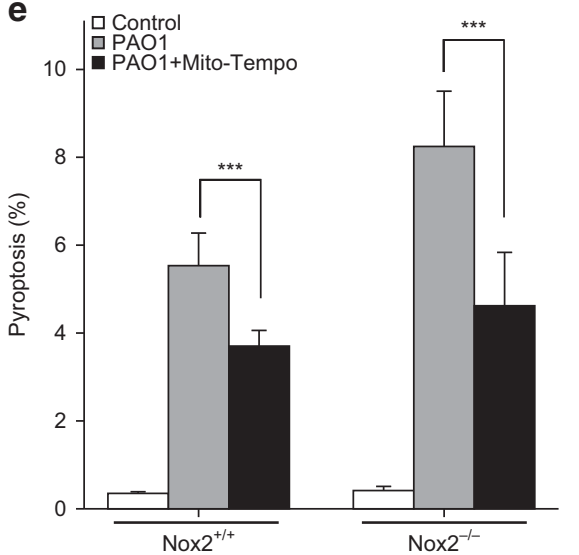

b

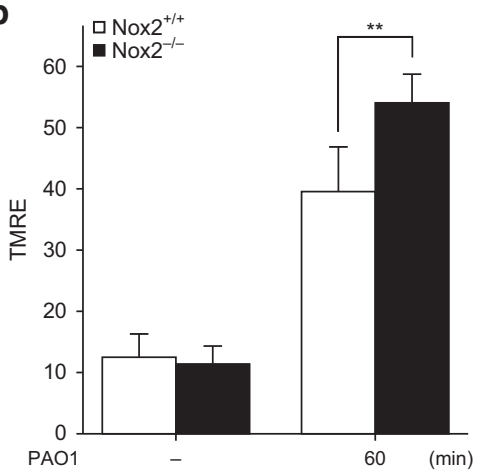

d

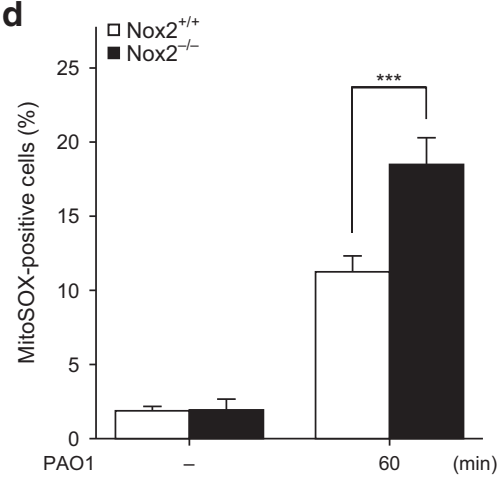

$\mathbf{g}$

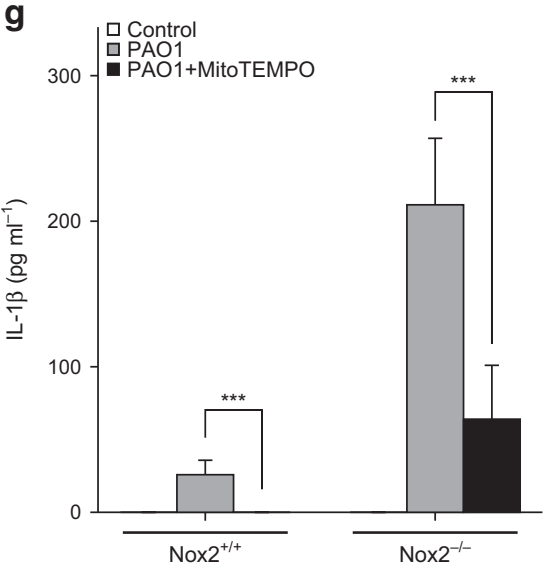

$\mathbf{f}$

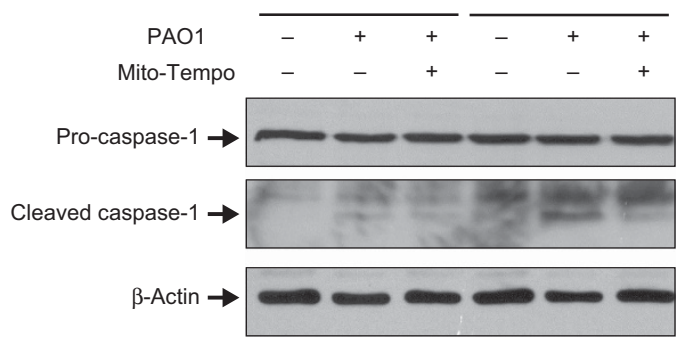

Figure 5 PAO1 infection damages mitochondria, generating mitochondrial reactive oxygen species (ROS) and inducing neutrophil pyroptosis. (a and b) The levels of tetramethylrhodamine ethyl ester (TMRE) and (c and d) mitochondrial ROS in bone marrow-derived neutrophils (BMDNs) from Nox2 ${ }^{-1}-$ and Nox2 $2^{+/+}$mice were determined 1 h after infection with PAO1 (multiplicity of infection (MOI) 20). (e) The levels of pyroptosis in BMDNs from Nox2 ${ }^{-/-}$ and Nox2 $2^{+/+}$mice were determined $1 \mathrm{~h}$ after infection with PAO1 (MOI 20) alone or with PAO1 in the presence of Mito-Tempo. (f and $\mathbf{g}$ ) BMDNs were infected with PAO1 (MOI 20) alone or PAO1 in the presence of Mito-Tempo; cell extracts and supernatants were harvested at $1 \mathrm{~h}$ postinfection. (f) Expression of cleaved caspase-1 in cell extracts was detected by western blotting. (g) The levels of interleukin (IL)-1 $\beta$ in the cell supernatants were detected by enzyme-linked immunosorbent assay. ${ }^{\star \star} P<0.01 ;{ }^{\star \star \star} P<0.001$. 
a

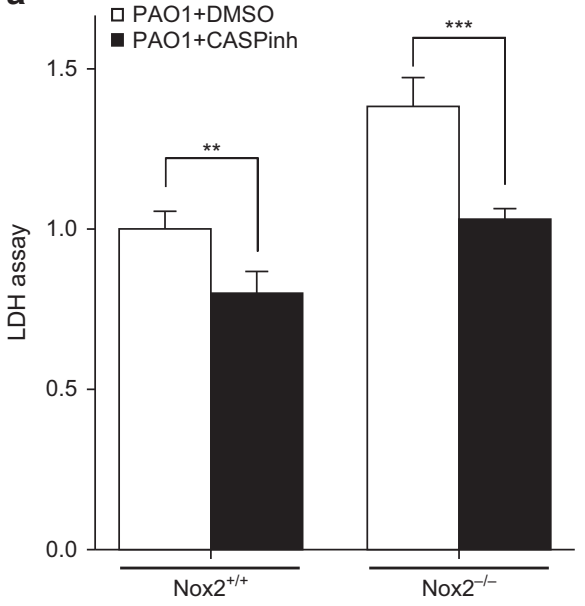

b

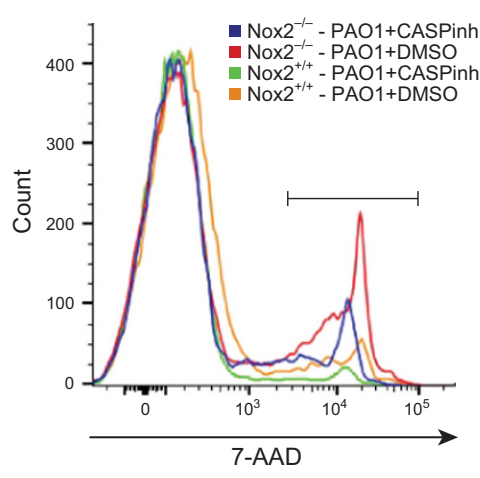

C

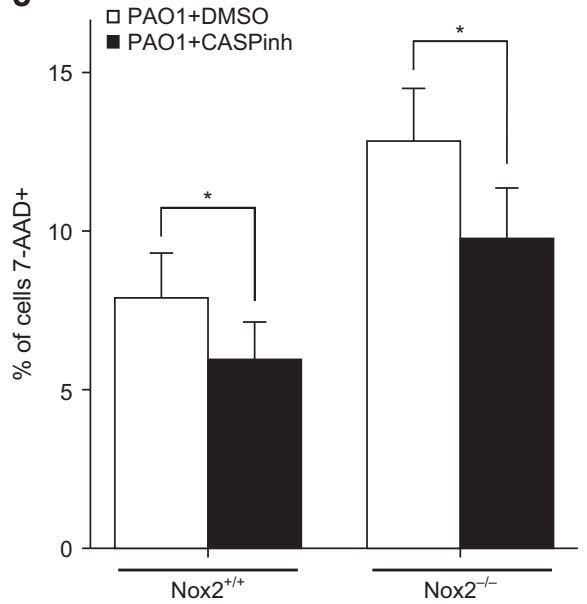

d

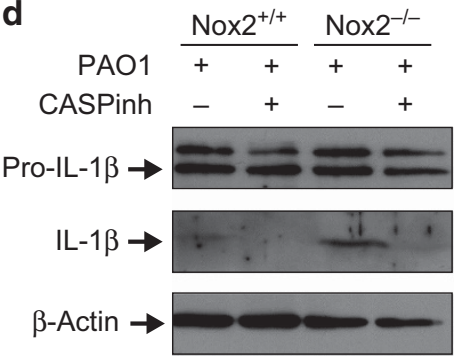

$\mathbf{e}$

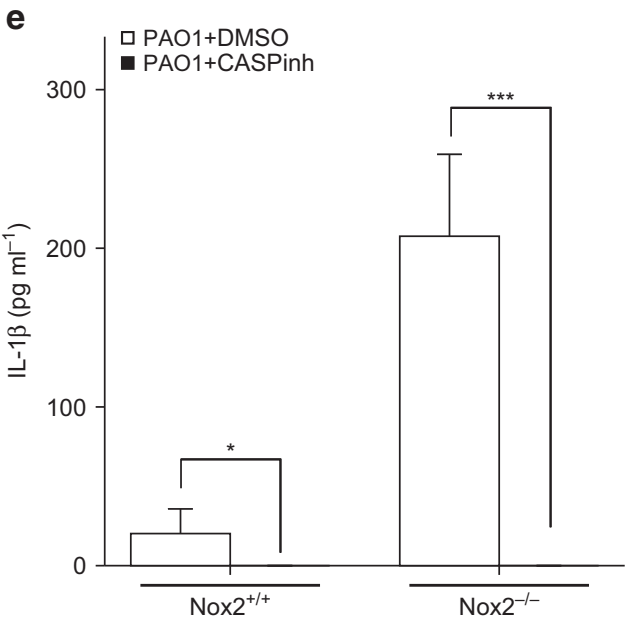

Figure 6 PAO1-infected neutrophils are responsible for both cell death and interleukin (IL)-1 $\beta$ secretion through caspase-1 (CASP1)-dependent

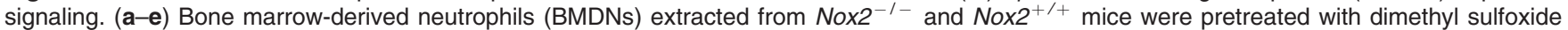
(DMSO) or the CASP1 inhibitor (CASPInh; AC-YVAD-CHO) 30 min prior to infection with green fluorescent protein (GFP)-tagged PAO1 (PAO1-GFP). (a) Cytoplasmic lactate dehydrogenase (LDH) release into the supernatant was quantified as a measure of pyroptosis. LDH release was measured at $1 \mathrm{~h}$ after infection with PAO1-GFP (multiplicity of infection (MOI) 20). (b) Representative flow cytometric plots and (c) the percentages of 7-AAD ${ }^{+}$BMDNs $^{-}$ were determined $1 \mathrm{~h}$ after infection with PAO1-GFP (MOI 20). (d) Expression of IL-1 $\beta$ in cell supernatants were detected by western blotting, relative to the expression of Pro IL-1 $\beta$ and the $\beta$-actin loading control in cell extracts. (e) The levels of IL-1 $\beta$ in cell supernatants were detected by enzyme-linked immunosorbent assay. ${ }^{\star} P<0.05 ;{ }^{\star \star} P<0.01 ;{ }^{* \star \star} P<0.001$. 7-AAD, 7-aminoactinomycin D.

\section{PA01-stimulated neutrophil pyroptosis depends on the NLRC4 inflammasome}

To know the role of NLRC4 in neutrophil pyroptosis in our system, we infected $\mathrm{Nlrc} 4^{+/+}$and $N \operatorname{lrc} 4^{-1-}$ BMDNs with two different doses of PAO1-GFP and evaluated each BMDN containing PAO1-GFP. The increase in pyroptosis of $\mathrm{Nlrc}^{+/+}$BMDNs containing PAO1-GFP was significantly decreased in Nlrc4 ${ }^{-1-}$ BMDNs (Figure 8a). However, the numbers of $\mathrm{Nlrc}^{-1-}$ BMDNs containing PAO1-GFP were not different than those of $N \operatorname{lrc} 4^{+/+}$BMDNs at both 10 and $60 \mathrm{~min}$ after infection (Figure 8b). The increased secretion of IL-1 $\beta$ in Nlrc $4^{+/+}$BMDNs was also significantly blunted in Nlrc4 ${ }^{-1-}$ BMDNs (Figure 8c). In contrast, the increase in pyroptosis and the number of BMDNs with PAO1GFP in $N \operatorname{lrp} 3^{+/+}$BMDNs were not changed in $N \operatorname{lrp} 3^{-/-}$
BMDNs (Figure 8d,e). However, increased secretion of IL-1 $\beta$ in Nlrp3 $3^{+/+}$BMDNs was significantly decreased in $N l r p 3^{-1-}$ BMDNs (Figure 8f).

\section{Toll-like receptor 5 (TLR5) contributes to neutrophil pyroptosis by regulating PAO1 uptake}

Given that, in addition to NLRC4, TLR5 is a known receptor for PAO1 flagellin, ${ }^{31,32}$ we wondered whether TLR5 is involved in PAO1-stimulated pyroptosis. The increase in pyroptosis in Tlr $5^{+/+}$BMDNs was slightly blunted in $T l r 5^{-/-}$BMDNs (Figure 9a). In contrast to the results obtained with $\mathrm{Nlrc4}^{-/-}$ BMDNs, fewer numbers of Tlr5 ${ }^{-1-}$ BMDNs with PAO1-GFP were observed compared with $T l r 5^{+/+}$BMDNs with PAO1GFP at both 10 and $60 \mathrm{~min}$ after infection (Figure 9b). These data suggest that the decreased pyroptosis in $\operatorname{Tl} 5^{-1-}$ 
a

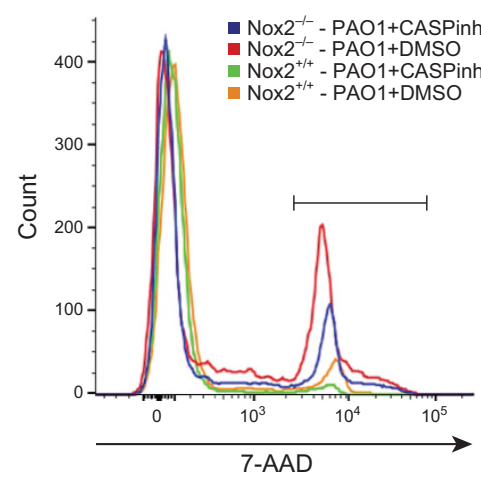

b

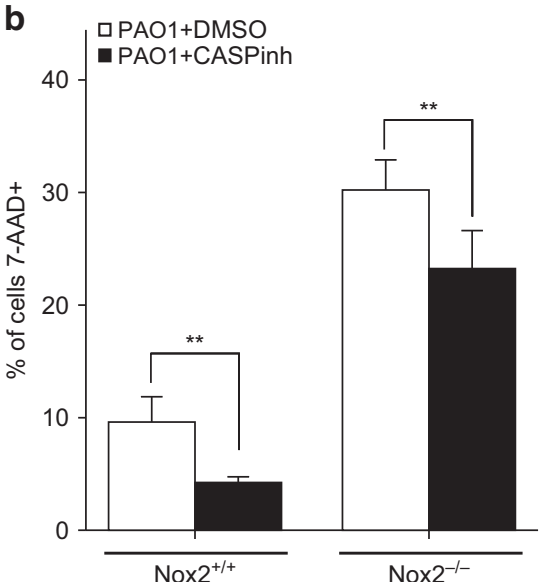

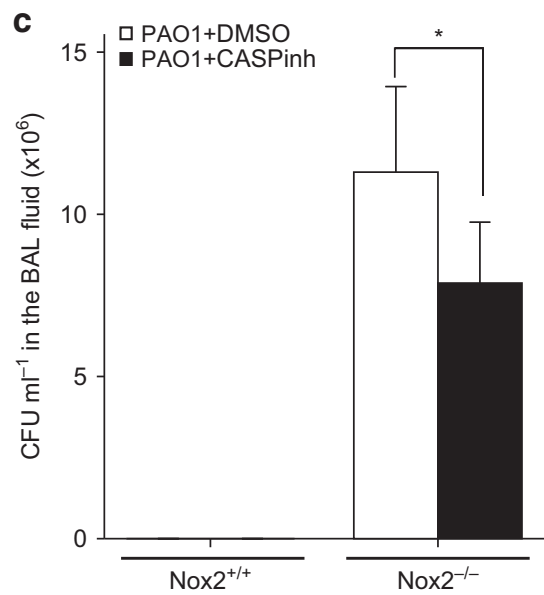

d
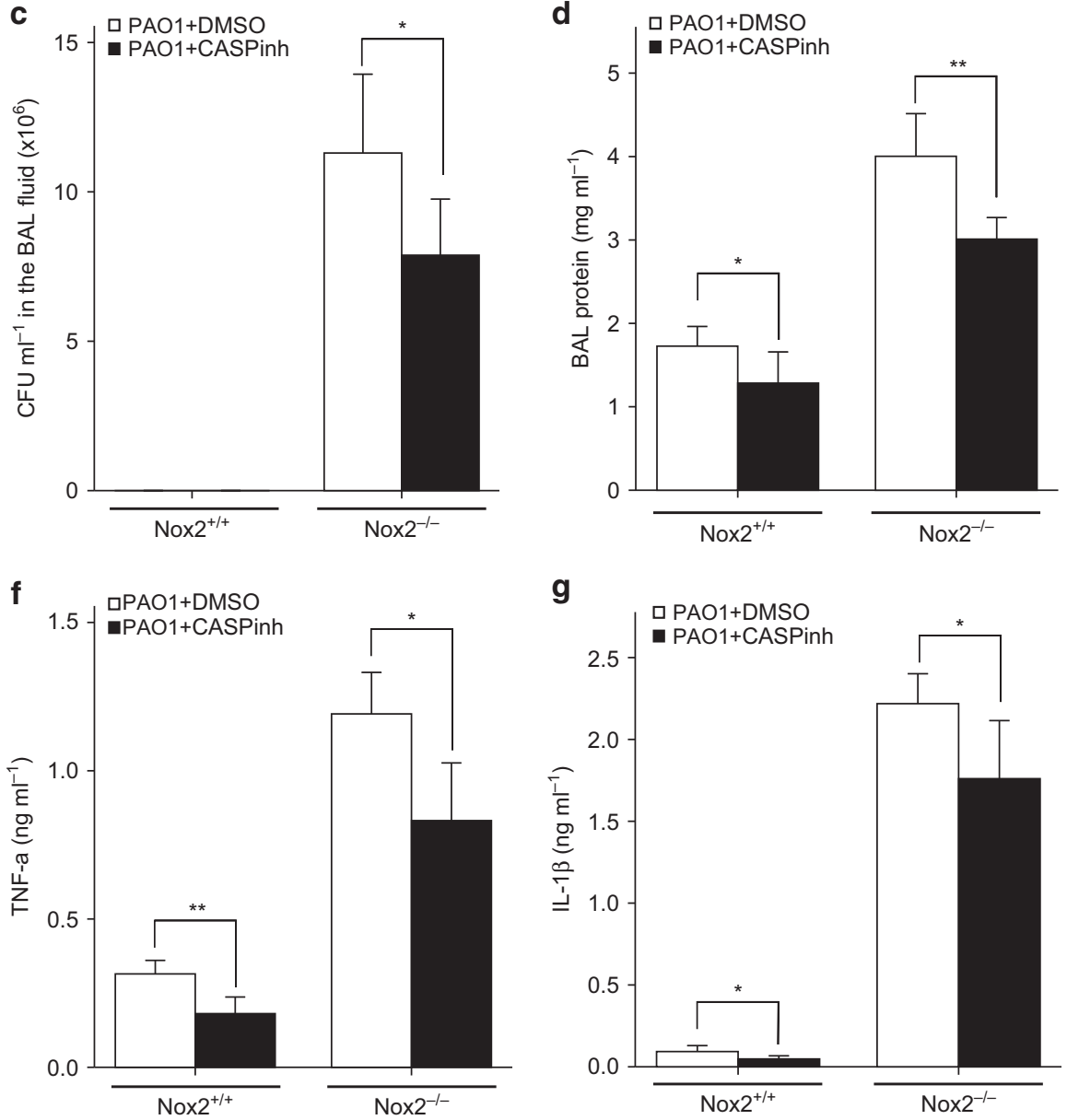

g

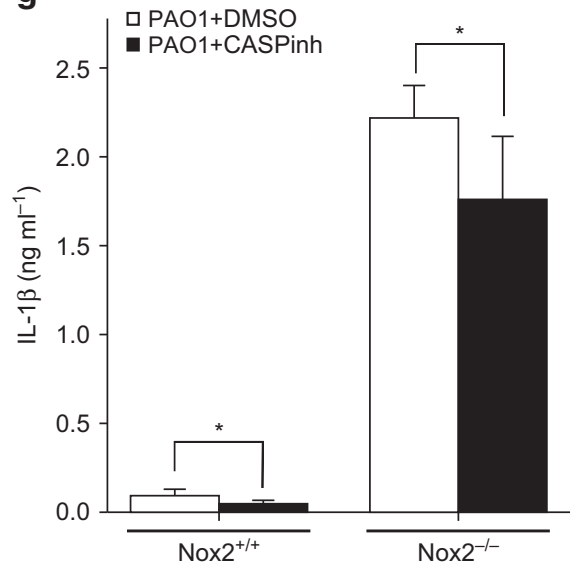

e

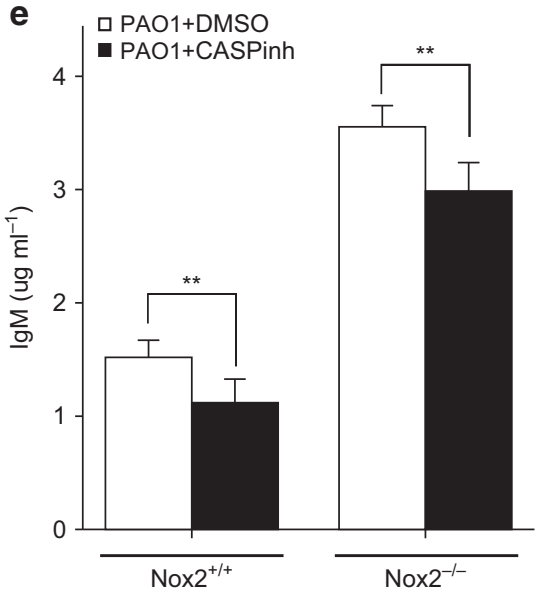

h

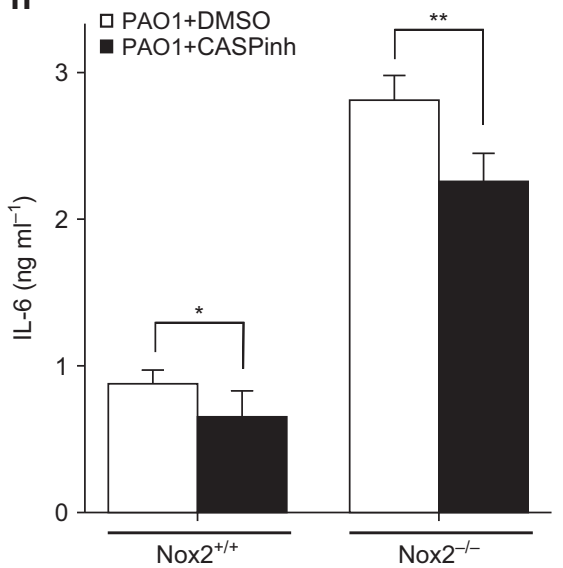

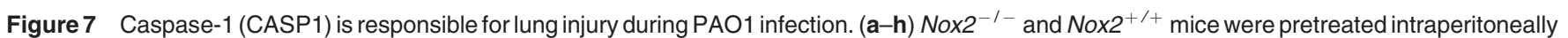
with DMSO or the CASP1 inhibitor (CASPInh; AC-YVAD-CHO) $1 \mathrm{~h}$ prior to intranasal instillation of $4 \times 10^{5}$ colony-forming units (CFU) of PAO1. (a) Representative flow cytometric plots and (b) the percentages of 7-AAD ${ }^{+}$neutrophils in the bronchoalveolar lavage (BAL) fluids from Nox2 ${ }^{-1-}$ and $\mathrm{Nox} 2^{+/+}$mice were determined at $24 \mathrm{~h}$ after intranasal instillation of PAO1. (c) Mice were killed at $24 \mathrm{~h}$ postinfection, and the bacterial burdens in the BAL fluid were measured. (d) Protein and (e) immunoglobulin M ( $\mathrm{IgM})$ levels in the BAL fluid from mice were determined at $24 \mathrm{~h}$ after intranasal instillation of PAO1. (f-h) Cytokine levels in BAL fluid after infection with PAO1. Levels of (f) tumor necrosis factor (TNF)- $\alpha$, (g) interleukin (IL)-1 $\beta$, and (h) IL-6 in the BAL fluids from mice were determined at $24 \mathrm{~h}$ after intranasal instillation of $\mathrm{PAO}$. ${ }^{\star} P<0.05$; ${ }^{\star \star} P<0.01$. DMSO, dimethyl sulfoxide; 7 -AAD, 7aminoactinomycin D.

BMDNs might be due to the decrease in initial uptake of PAO1GFP in Tlr5 $5^{-1-}$ BMDNs. Moreover, the increase in IL-1 $\beta$ secretion in $T l r 5^{+/+}$BMDNs was dramatically decreased in Tlr5 ${ }^{-1-}$ BMDNs (Figure 9c).
PA01 flagellin is required for neutrophil pyroptosis upon infection

As we found that both NLRC4 and TLR5 are involved in PAO1stimulated neutrophil pyroptosis, we hypothesized that PAO1 
a
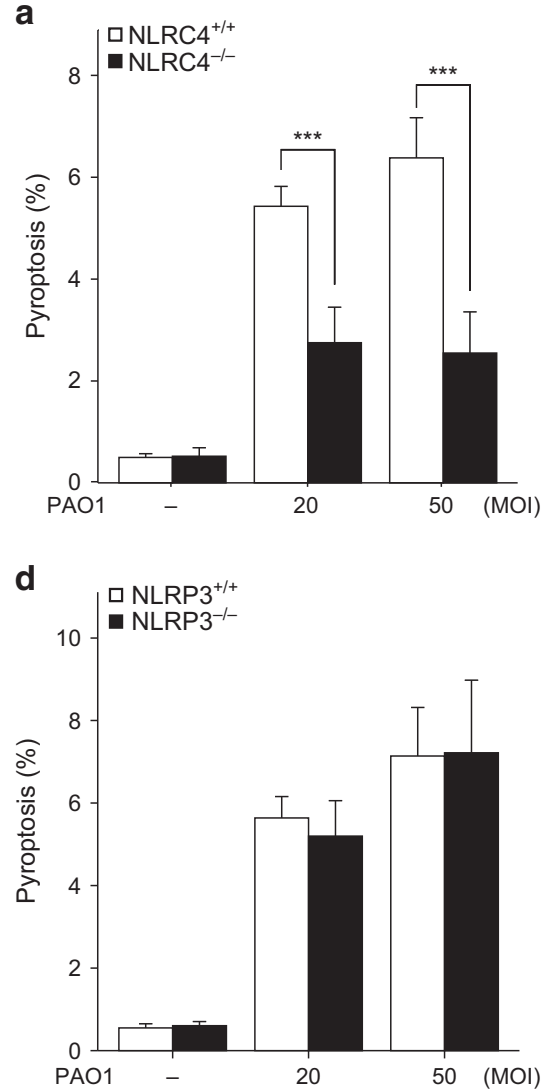

b

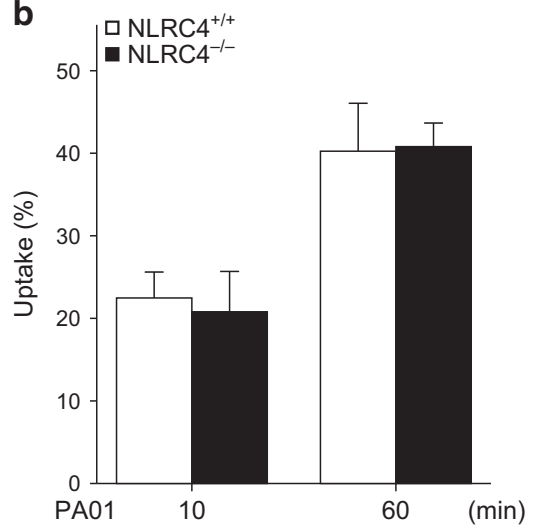

e

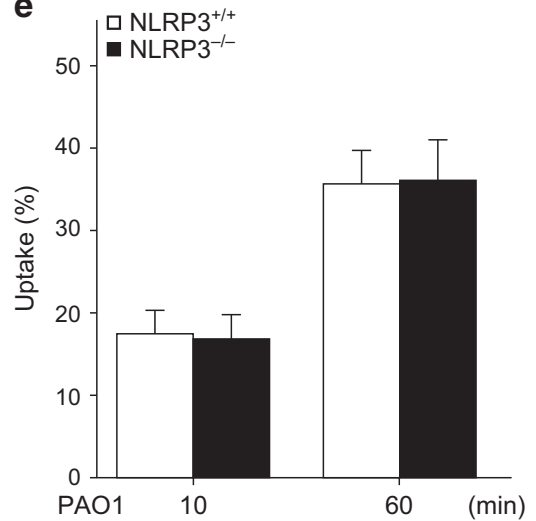

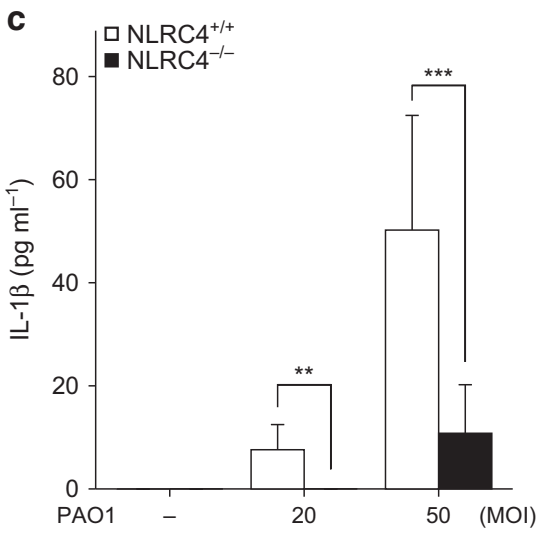

f

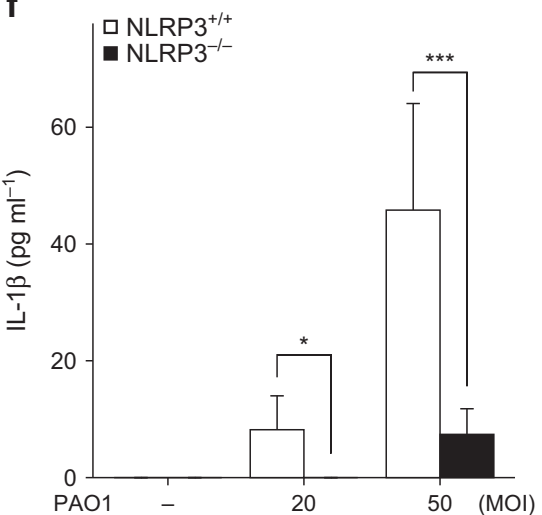

Figure 8 Neutrophil pyroptosis induced by PAO1 infection is mediated by the Nod-like receptor family, CARD domain-containing 4 (NLRC4)

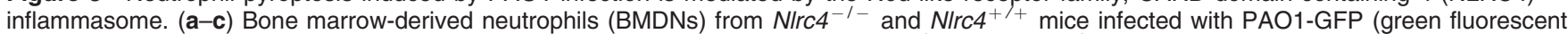
protein). (a) The percentages of pyroptotic BMDNs, including PAO1-GFP, from N/rc4 ${ }^{-1-}$ and $\mathrm{N} / \mathrm{rc}^{+/+}$mice were determined $1 \mathrm{~h}$ after infection with PAO1-GFP (multiplicity of infection (MOI) 20,50). (b) The percentages of BMDN uptake from NIrc4 ${ }^{-1-}$ and N/rc4 ${ }^{+/+}$mice were determined at 10 and 60 min after infection with PAO1-GFP (MOI 20). (c) Interleukin (IL)-1 $\beta$ levels were detected in the supernatants of BMDNs from NIrc4 ${ }^{-1-}$ and NIrc4 ${ }^{+/+}$ mice after infection with PAO1 (MOI 20,50). (d-f) BMDNs from NIrp3 ${ }^{-1-}$ and NIrp3 ${ }^{+/+}$mice infected with PAO1-GFP. (d) The percentages of pyroptotic BMDNs, including PAO1-GFP, from N/rp3 ${ }^{-/-}$and $\mathrm{NIrp3}^{+/+}$mice were determined 1 h after infection with PAO1-GFP (MOI 20,50). (e) The percentages of BMDN uptake from NIrp3 $3^{-1-}$ and N/rp3 ${ }^{+/+}$mice were determined at 10 and 60 min postinfection with PAO1-GFP (MOI 20). (f) IL-1 $\beta$ levels were detected in the supernatants of BMDNs from NIrp3 ${ }^{-1-}$ and $N / r p 3^{+/+}$mice $1 \mathrm{~h}$ after infection with PAO1 $(\mathrm{MOI} 20,50) .{ }^{*} P<0.05 ;{ }^{* \star} P<0.01 ; *^{* \star} P<0.001$.

a

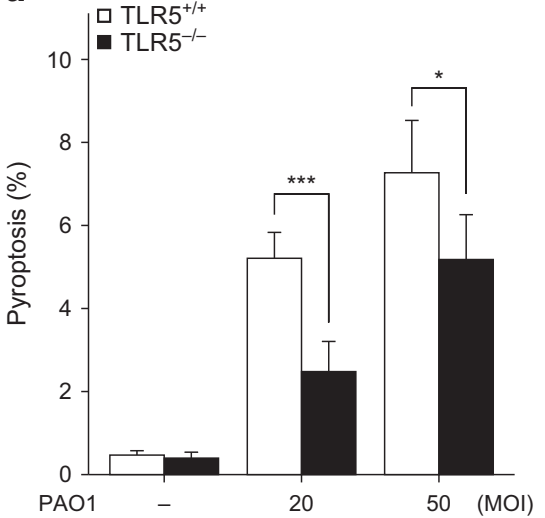

b

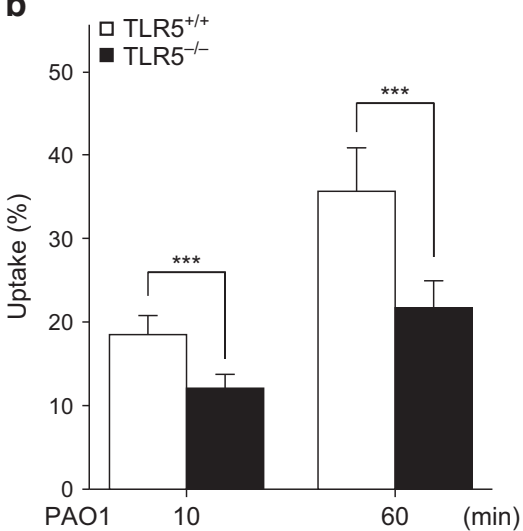

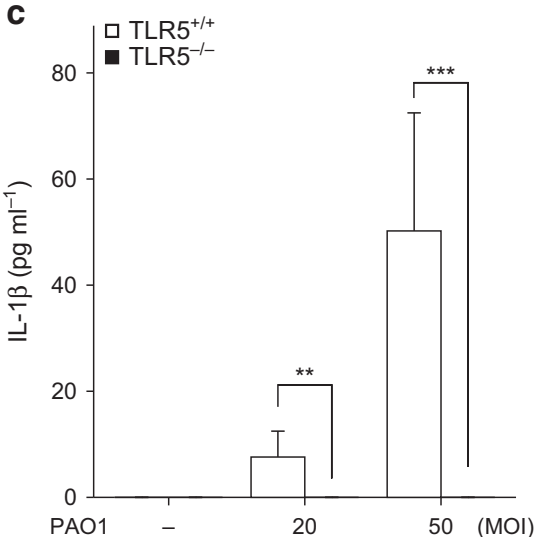

Figure 9 Toll-like receptor 5 (TLR5) contributes to PAO1-mediated induction of neutrophil pyroptosis. (a-c) Bone marrow-derived neutrophils (BMDNs) from TIr5 $5^{-1-}$ and Tlr5 $5^{+1+}$ mice were infected with PAO1-GFP. (a) The percentages of pyroptotic BMDNs, including PAO1-GFP (green fluorescent protein), from Tlr5 $5^{-/-}$and TIr $5^{+/+}$mice were determined $1 \mathrm{~h}$ after infection with PAO1-GFP (multiplicity of infection (MOI) 20, 50). (b) The percentages of BMDN uptake from TIr5 ${ }^{-1-}$ and TIr5 ${ }^{+/+}$mice were determined at 10 and 60 min after infection with PAO1-GFP (MOI 20). (c) Interleukin (IL)-1 $\beta$ levels were detected in the supernatants of BMDNs from Tlr5 ${ }^{-1-}$ and $T / r 5^{+/+}$mice $1 \mathrm{~h}$ after infection with PAO1 (MOI 20, 50). ${ }^{*} P<0.05$;

${ }^{* *} P<0.01 ;{ }^{* * *} P<0.001$ 

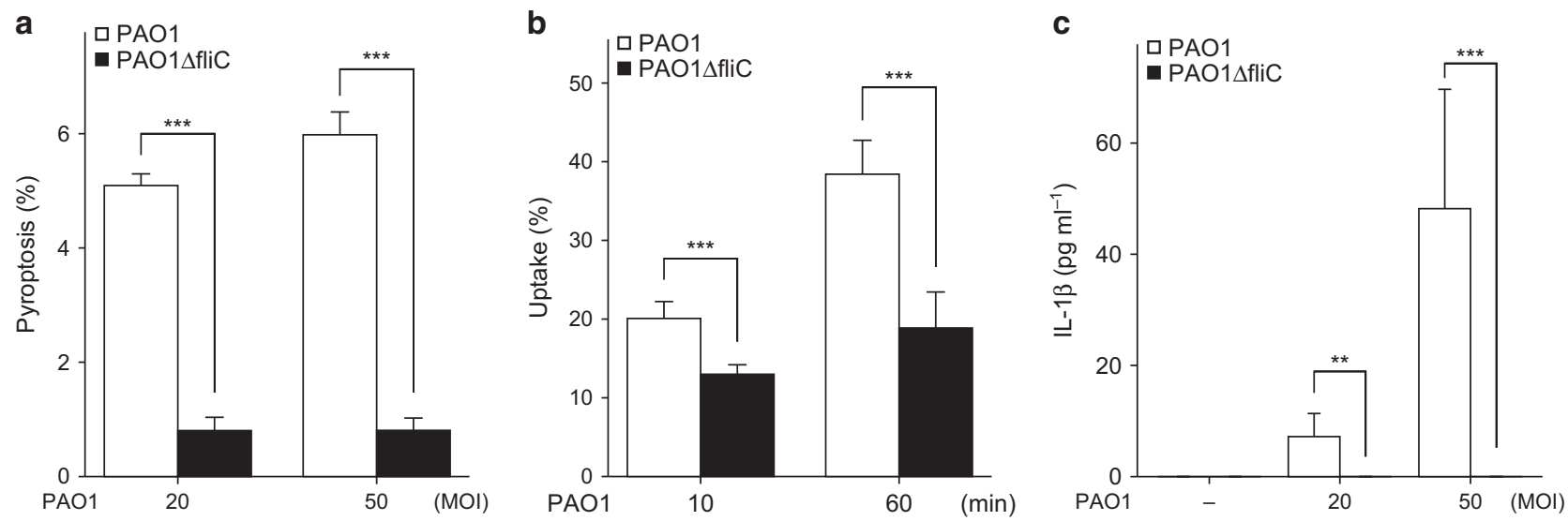

Figure 10 PAO1 flagellin is required for neutrophil pyroptosis upon PAO1 infection. (a-c) Bone marrow-derived neutrophils (BMDNs) from wild-type (WT) mice were infected with PAO1-GFP (green fluorescent protein) and PAO1 $\Delta$ fliC (a PAO1 mutant devoid of flagellin production)-GFP. (a) The percentages of pyroptotic BMDNs, including PAO1-GFP, from WT mice were determined $1 \mathrm{~h}$ after infection with PAO1-GFP and PAO1 $\triangle$ fliC-GFP (multiplicity of infection (MOI) 20,50). (b) The percentages of BMDN uptake from WT mice were determined at 10 and 60 min after infection with PAO1GFP and PAO1 $\triangle$ fliC-GFP (MOI 20). (c) Interleukin (IL)-1 $\beta$ levels were detected in the supernatants of BMDNs from WT mice $1 \mathrm{~h}$ after infection with PAO1GFP and PAO1 1 fliC-GFP (MOI 20, 50). ${ }^{\star \star} P<0.01 ;{ }^{* \star \star} P<0.001$.

flagellin is responsible for the induction of neutrophil pyroptosis upon infection. We observed that neutrophils infected with a PAO1 mutant devoid of flagellin production $(\mathrm{PAO} 1 \Delta \mathrm{fliC})$ exhibited significantly decreased pyroptosis compared with neutrophils infected with WT PAO1 (Figure 10a). The number of BMDNs with PAO1 $\triangle$ fliC-GFP was also lower than the number of BMDNs infected with PAO1-GFP (Figure 10b). Moreover, the level of IL- $1 \beta$ secretion induced by PAO1 $\Delta$ FliC infection was much lower than that induced by PAO1 infection (Figure 10c). As the $P$. aeruginosa type 3 secretion system (T3SS) is responsible for NLRC4 inflammasome activation in macrophages, ${ }^{20}$ we determined the extent of neutrophil pyroptosis and pathogen uptake upon infection with PAO1 $\triangle T$ T3SS-GFP. The levels of pyroptosis and neutrophil uptake in the context of PAO1 $\Delta$ T3SS-GFP infection were not significantly different than those in the context of PAO1-GFP infection (Supplementary Figure S6a,b), while the level of IL$1 \beta$ secretion stimulated by PAO1 $\Delta \mathrm{T} 3 \mathrm{SS}$ infection was much lower than that stimulated by PAO1 infection (Supplementary Figure S6c).

PAO1 flagellin is responsible for neutrophil pyroptosis and lung injury during acute lung infection

To investigate the physiological role of PAO1 flagellin in our acute lung infection mouse model, we challenged $\mathrm{Nox}^{+/+}$or Nox $2^{-1-}$ mice with PAO1 or PAO1 $\Delta$ fliC. In Nox2 $2^{+/+}$mice, the percentage of pyroptotic neutrophils was extremely low (about 1\%) upon infection with either PAO1 or PAO1 $\Delta$ fliC, while the PAO1-stimulated increase in pyroptosis in $\mathrm{Nox}^{-1-}$ mice (about 10\%) was significantly decreased upon PAO1 $\Delta$ fliC infection (about 6\%) (Figure 11a). The increased PAO1 CFUs in the BAL fluid of Nox2 $2^{-1-}$ mice was also decreased by using $\mathrm{PAO} 1 \Delta$ fliC (Figure 11b). In accordance with the lower bacterial burden of PAO1 $\Delta$ fliC, the increased levels of lung injury markers such as BAL protein (Figure 11c) and IgM (Figure 11d) in $\mathrm{Nox}^{-1-}$ mice induced by PAO1 infection were blunted in the context of PAO1 $\Delta$ fliC infection. Lower levels of inflammatory cytokines such as TNF- $\alpha$ (Figure 11e), IL-1 $\beta$ (Figure 11f), and IL-18 (Figure 11g) were observed in Nox $2^{-1-}$ mice infected with PAO1 $\Delta$ fliC than in mice infected with PAO1.

\section{PA01 flagellin mediates pyroptosis in infected human neutrophils}

To investigate whether our in vivo and in vivo results were mirrored in human neutrophils, we investigated whether PAO1 flagellin mediated pyroptosis in human neutrophils. To this end, we infected human neutrophils with PAO1 or PAO1 $\Delta$ fliC and quantified pyroptosis at $60 \mathrm{~min}$ postinfection. Neutrophils infected with PAO1 $\Delta$ fliC exhibited significantly decreased pyroptosis compared with neutrophils infected with PAO1 (Figure 12a). In contrast, the number of human neutrophils with PAO1-GFP was not significantly different than that with $\mathrm{PAO} 1 \Delta$ fliC-GFP (Figure 12b). Furthermore, PAO $1 \Delta \mathrm{fliC}$ infection resulted in reduced IL-1 $\beta$ and IL-18 secretion compared with PAO1 infection (Figure 12c,d). We also used time-lapse video microscopy to track pyroptosis in live human neutrophils infected with either PAO1-GFP or PAO1 $\Delta$ fliC-GFP (data not shown). Cleaved CASP1 (red) started to appear in human neutrophils throughout the cytoplasm at $20 \mathrm{~min}$ after PAO1 infection. The infected neutrophils had a swollen appearance at $40 \mathrm{~min}$ and underwent cell death by $60 \mathrm{~min}$ after PAO1 infection. In contrast, cleaved CASP1 and cell death were barely observed in neutrophils infected with PAO1 $\Delta$ fliC-GFP (Figure 12b, video clip). These results indicate that flagellin is responsible for pyroptosis in human neutrophils upon PAO1 infection.

\section{DISCUSSION}

Neutrophil pyroptosis is activated by acute $P$. aeruginosa infection via inflammasome signaling. Flagellin within 
a

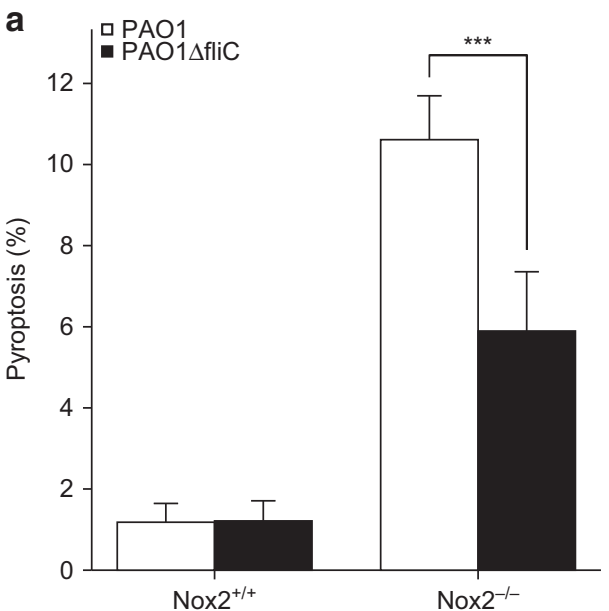

C

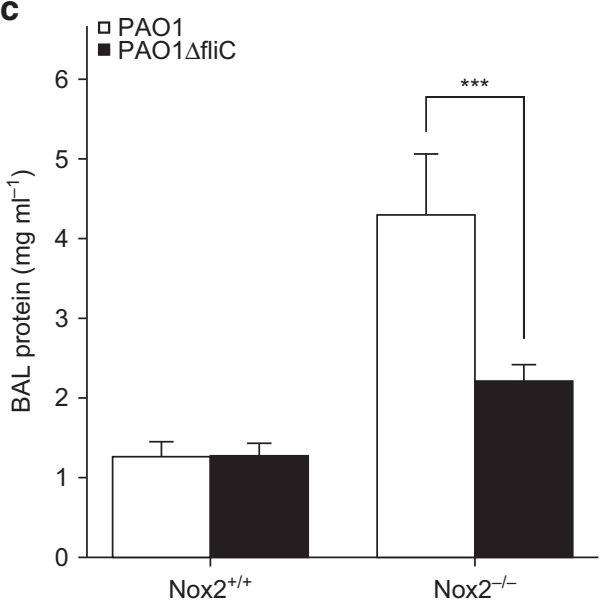

e

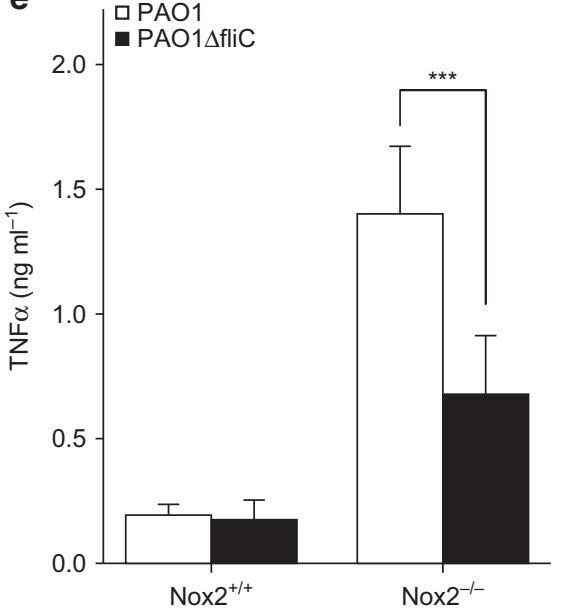

f

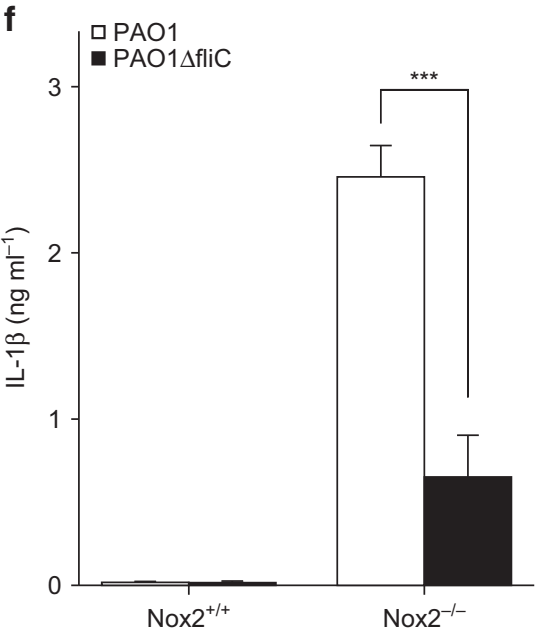

b

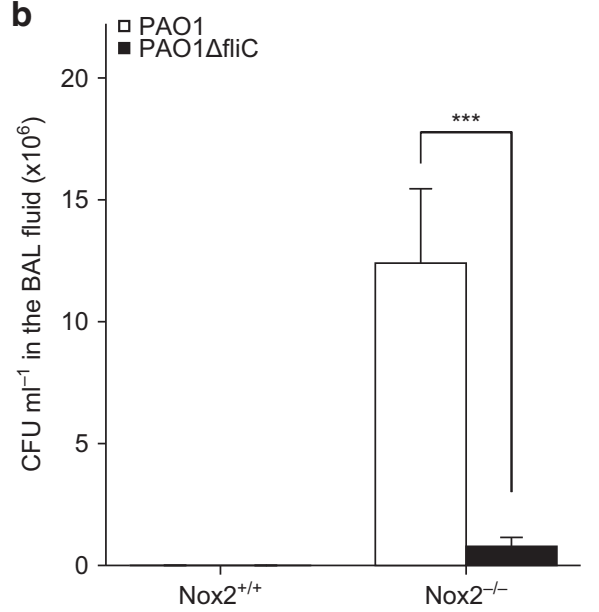

d

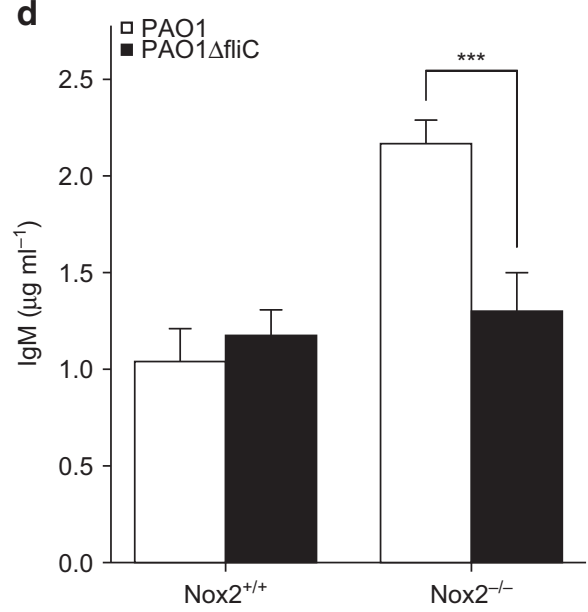

g

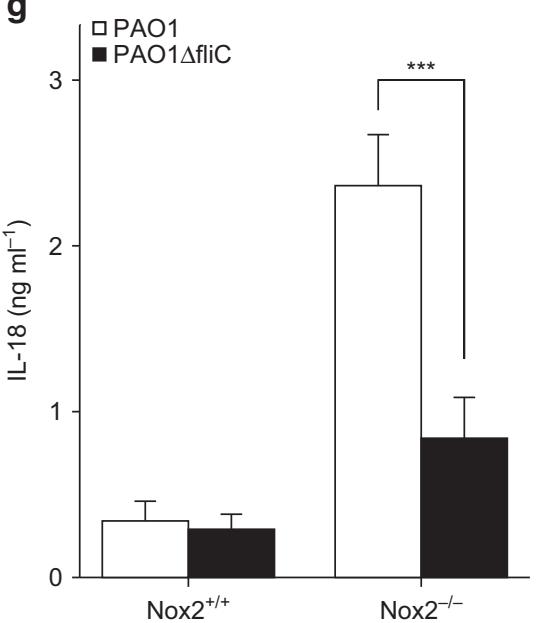

Figure 11 PAO1 flagellin is responsible for lung injury during PAO1 infection. (a-g) Mice were instilled intranasally with $4 \times 10^{5}$ colony-forming units (CFU) of PAO1 or a PAO1 mutant devoid of flagellin production (PAO1 $\triangle \mathrm{fliC}$ ). (a) The percentages of pyroptotic neutrophils in the bronchoalveolar lavage

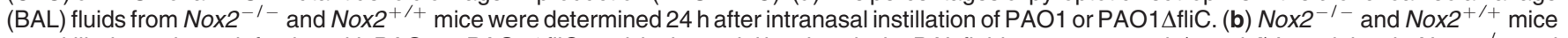
were killed at $24 \mathrm{~h}$ postinfection with PAO1 or PAO1 $\triangle \mathrm{fliC}$, and the bacterial burdens in the BAL fluid were measured. (c and d) Lung injury in Nox2 ${ }^{-1-}$ and Nox2 ${ }^{+/+}$mice after infection with PAO1 or PAO1 $\triangle$ fliC. (c) Protein and (d) immunoglobulin M (IgM) levels in the BAL fluid from WT mice were determined $24 \mathrm{~h}$ after infection with PAO1 or PAO1 $\Delta$ fliC. $(\mathbf{e}-\mathbf{g})$ Cytokine levels in BAL fluid after infection with PAO1 or PAO1 $\Delta$ fliC. The levels of (e) tumor necrosis factor (TNF)- $\alpha$, (f) interleukin (IL)-1 $\beta$, and (g) IL-18 in the BAL fluids from Nox2 $2^{-1-}$ and Nox2 $2^{+/+}$mice were determined $24 \mathrm{~h}$ after intranasal instillation of PAO1 or PAO1 $\Delta$ fliC. ${ }^{* \star *} P<0.001$. 

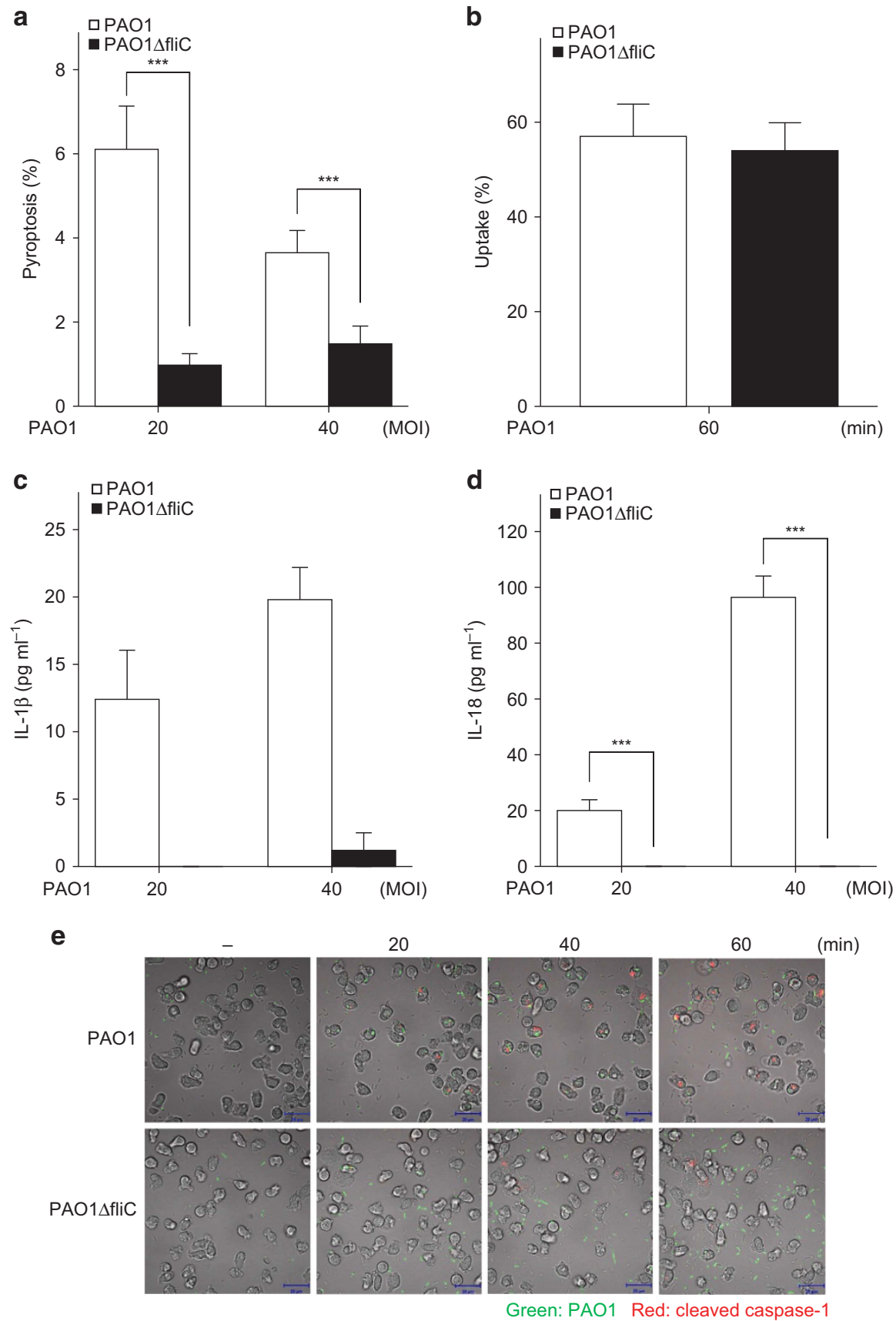

Figure 12 PAO1 flagellin induces pyroptosis in infected human neutrophils. (a-d) Human neutrophils were infected with PAO1-GFP (green fluorescent protein) or PAO1 $\triangle$ fliC (a PAO1 mutant devoid of flagellin production)-GFP. (a) The percentages of pyroptotic human neutrophils, including PAO1-GFP, were determined $1 \mathrm{~h}$ after infection with PAO1-GFP or PAO1 $\triangle$ fliC-GFP (multiplicity of infection (MOI) 20,40). (b) The percentages of human neutrophils with PAO1-GFP or PAO1 $\triangle$ fliC-GFP uptake were determined $1 \mathrm{~h}$ after infection (MOI 20). (c) Interleukin (IL)-1 $\beta$ and (d) IL-18 levels were detected in the supernatants of human neutrophils $1 \mathrm{~h}$ after infection with PAO1-GFP or PAO1 $\triangle$ fliC-GFP $(\mathrm{MOI} 20,40)$. (e) A series of images from a representative livecell video microscopic experiment showing human neutrophils, PAO1, and caspase-1 (CASP1) activity during uptake and after uptake. PAO1 continued to grow within human neutrophils, which can result in cell lysis. Active CASP1 was detected with the far-red FLICA-660 probe, and cells were infected with GFP-tagged PAO1 or GFP-tagged PAO1 $\Delta$ fliC. ${ }^{* \star *} P<0.001$.

P. aeruginosa and mitochondrial ROS are responsible for neutrophil pyroptosis upon acute lung infection through CASP1-dependent signaling in Nox $2^{-1-}$ mice, leading to greater lung inflammation and injury as a consequence of increased $P$. aeruginosa burden in the lungs (Supplementary Figure S7). Recently, NLRC4-dependent inflammasome activation in neutrophils, rather than macrophages, was shown to be responsible for Salmonella clearance after challenge, ${ }^{23}$ supporting our results that neutrophils have a critical role in host protection. However, in this report, host protection achieved by neutrophils is mediated by NLRC4/ CASP1-dependent IL-1 $\beta$ secretion, but not pyroptosis. ${ }^{23}$ 
Although macrophage pyroptosis is essential for host protection because it releases pathogens from macrophages into the extracellular environment, the role of neutrophil pyroptosis in host protection is controversial. If neutrophil-ingested pathogens can resist typical neutrophil antimicrobial responses, such as the generation of ROS and degranulation, the absence of neutrophil pyroptosis may ultimately provide a niche for survival. Indeed, several studies have reported that Salmonella, ${ }^{33}$ Staphylococcus aureus, ${ }^{34}$ Nesisseria gonorrhoeae, ${ }^{35}$ Chlamydia pneumonia, ${ }^{36}$ and Anaplasma phagocytophilum ${ }^{37}$ all replicate efficiently within neutrophils, thereby avoiding the immune system. In our study, an almost negligible amount of pyroptotic neutrophils (about $0.7 \%$ of the total neutrophils in the bronchoalveolar space) was observed during PAO1 lung infection. Interestingly, neutrophil pyroptosis was dramatically increased in Nox2 $2^{-1-}$ mice upon infection (about $11 \%$ of the total neutrophils in the bronchoalveolar space). The increased pyroptosis in neutrophils devoid of antimicrobial activity suggests that high pathogen burdens induce pyroptosis in neutrophils. In vitro studies using $P$. aeruginosa-infected macrophages have revealed that the inflammasome complex, including NLRC4, CASP1, and ASC, is essential to IL- $1 \beta$ production. ${ }^{38-41}$ In vivo lung infection models investigating $P$. aeruginosa have also shown that NLRC4/CASP1 signaling pathways in macrophages are critical to IL- $1 \beta$ production. ${ }^{20,21}$ Although macrophages are typically assumed to be the major contributors to IL- $1 \beta$ generation and secretion, recent studies have reported that IL- $1 \beta$ production is apparent in human and murine neutrophils under infectious conditions. ${ }^{23,42-44}$ However, the physiological role and regulatory mechanisms by which neutrophils stimulate IL-1 $\beta$ production are not clear. Recently, researchers have shown that neutrophils are recruited to infection sites and are responsible for ASC-independent and CASP1-dependent IL- $1 \beta$ production in acute pneumonia and peritonitis infectious models with $P$. aeruginosa. ${ }^{25}$ These results support our data, which suggest that IL-1 $\beta$ production in neutrophils is dependent on CASP1 signaling pathway in in vitro and in vivo models of $P$. aeruginosa infection under NOX2-deficient conditions. Nevertheless, we were unable to demonstrate a direct physiological role in IL- $1 \beta$ production in the same condition.

Cytosolic bacterial flagellin in intracellular pathogens such as S. typhimurim and L. pneumophila is the major trigger of the host immune response; this signaling occurs through the NLRC4 inflammasome. ${ }^{6,11,12,45}$ NLRC4 is critical for inflammasome activation against extracellular pathogens, such as P. aeruginosa. ${ }^{39,40}$ However, in vitro studies using macrophages have demonstrated that $P$. aeruginosa flagellin is not required to activate the NLRC4/CASP1 inflammasome, even though proteins secreted through the type III secretion system are critical for inducing CASP1 activation via NLRC4. ${ }^{41}$ In contrast, during acute lung infection, $P$. aeruginosa flagellin is responsible for host pathological damage via CASP1 activation and IL-1 $\beta$ secretion in macrophages. ${ }^{21}$ Although the immunological function and regulatory mechanism of P. aeruginosa flagellin is well defined in macrophages, its role in host immunity in neutrophils has not yet been clearly defined. Here we demonstrated a critical role of PAO1 flagellin for CASP1-dependent inflammasome activation in neutrophils by showing that the increases in secretion of IL-1 $\beta$ and IL18, neutrophil pyroptosis, and lung injury in infected Nox $2^{-1-}$ mice were all significantly attenuated by infection with $\mathrm{PAO} 1 \Delta \mathrm{fliC}$. Our results reveal a novel regulatory mechanism in neutrophils by which $P$. aeruginosa flagellin is the main component for inflammasome activation and has broad relevance to imflammasome-driven immunity and pathology.

However, we could not determine the precise role of NLRC4 in inflammasome activation and host physiology in the absence of NOX2 during PAO1 lung infection. Instead, we founded that NLRC4 induces inflammasome activation in neutrophils during PAO1 infection. In support of this finding, we showed that the increased levels of pyroptosis and IL- $1 \beta$ secretion observed in PAO1-infected WT BMDNs were blunted in PAO1-infected Nlrc4 ${ }^{-1-}$ BMDNs. However, interestingly, the increased level of pyroptosis observed in PAO1-infected WT BMDNs was also observed in infected Nlrp3 $3^{-1-}$ BMDNs, while the increase in IL-1 $\beta$ secretion was blunted in Nlrp $3^{-1-}$ BMDNs. Though several reports indicate that the NLRP3 inflammasome is involved in IL-1 $\beta$ processing in neutrophils, ${ }^{42,44,46}$ the precise reasons why NLRP3 in neutrophil is responsible for IL-1 $\beta$ processing, but not pyroptosis, in our study remain to be further investigated. However, given that murine CASP11 promotes IL-1 $\beta$ processing via the NLRP3-CASP1 signaling pathway in a manner independent of NLRC4-CASP1 signaling, ${ }^{47}$ signaling pathway upstream of NLRP3 such as CASP11 may activate NLRP3-mediated IL-1 $\beta$ secretion, independent of NLRC4CASP1 signaling, in infected neutrophils.

In vivo neutrophil-depletion studies replicated the outcome phenotype for the condition of decreased neutrophils in Nox $2^{+/+}$mice upon PAO1 lung infection, in which increased bacterial burden, injury, inflammation, and IL-1 $\beta / \mathrm{IL}-18$ secretion were observed.

Interestingly, in PAO1-infected WT mouse, IL-18 secretion increased when both macrophages and neutrophils were present, compared with when macrophages were depleted. IL-18 secretion was further increased when neutrophils were depleted (Figure 2i). Given studies have shown that $P$. aeruginosa activates NLRC4 inflammasomes leading to IL18 production and regulated recruitment of neutrophils to clear the pathogen, ${ }^{6,38,48}$ these results suggest that, in PAO1-infected WT mice, NLRC4 activation in neutrophils is suppressed and NLRC4 activation in macrophages induces IL-18 secretion, while

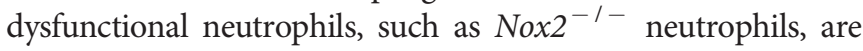
activated through NLRC4 and/or NLRP3 inflammasomes, leading to greater IL-18 production and neutrophil pyroptosis.

$P$. aeruginosa has been shown to activate NLRP3 inflammasomes and IL- $1 \beta$ processing through induction of mitochondrial $\mathrm{Ca}^{2+}$ release in IB3-1 cells, human bronchial epithelial cells. ${ }^{49}$ Another report demonstrated that $P$. aeruginosa activates NLRC4 inflammasomes and IL- $1 \beta$ processing by inducing mitochondrial ROS and DNA release in macrophages. ${ }^{50}$ Given that, in the present study, PAO1-mediated 
increases in IL-1 $\beta$ secretion and pyroptosis in Nox $2^{-1-}$ BMDNs were significantly diminished in the presence of MitoTempo, a mitochondrial ROS scavenging reagent, we suggest that mitochondrial ROS might act as a bridge between NOX2mediated signaling and inflammasome activation in neutrophils activated by PAO1 infection.

Although it is well known that $P$. aeruginosa flagellin is recognized by TLR5 in addition to NLRC4, ${ }^{32}$ TLR5 is not required for CASP1 activation in response to $P$. aeruginosa. Moreover, Tlr $5^{-1-}$ macrophages still demonstrated IL-1 $\beta$ secretion and cell death. ${ }^{39}$ To date, the precise role of TLR5 in inflammasome activation in $P$. aeruginosa-infected neutrophils has not yet been defined. However, TLR5 was shown to be highly expressed in infiltrated neutrophils in two human lung diseases commonly associated with chronic $P$. aeruginosa infection, cystic fibrosis, and bronchiectasis. ${ }^{51}$ In addition, TLR5 is stored intracellularly in neutrophils and translocated to the cell surface through cooperative TLR1 and TLR2 signaling. ${ }^{51}$ This report supports our findings that TLR5 is responsible for PAO1-induced neutrophil pyroptosis, which is based on our experiments using $T l r 5^{+/+}$and $T l r 5^{-1-}$ BMDNs. As the extent of pyroptosis reduction achieved with $\mathrm{PAO} \Delta \mathrm{FliC}$ infection was almost equal to the sum of the extent of pyroptosis reduction in infected Nlrc4 $4^{-1-}$ BMDNs and the extent of reduction in infected $T \mathrm{Tr} 5^{-1-}$ BMDNs, PAO1induced neutrophil pyroptosis might be triggered by cooperative recognition of PAO1 flagellin by TLR5 and NLRC4. However, although TLR5 is clearly responsible for PAO1 uptake by infected neutrophils, it remains the scope of future studies to determine whether intracellular TLR5 is directly involved in PAO1-induced neutrophil pyroptosis.

Our study demonstrated previously unknown mechanisms of neutrophil pyroptosis during acute lung infection with $P$. aeruginosa and shed light on the pathological role of this process. Thus inhibitors that specifically target neutrophil pyroptosis are likely to be useful for curing highly intractable $P$. aeruginosa infections most commonly seen in patients with neutrophil function disorders.

\section{METHODS}

Animals. Male mice between 8 and 12 weeks of age were used in all experiments. Nlrc4 ${ }^{-1-}$ mice on the C57BL/6 background were kindly provided by Professor Jong-Hwan Park (Chonnam National University, Gwangju, Korea). C57BL/6 mice were purchased from Orient Bio (Gyeonggi, Korea), whereas Nox2 $2^{-1-}, N \operatorname{lrp} 3^{-1-}$, and Tlr5 $5^{-1-}$ mice on a C57BL/6 background were bred at the animal facility of Yonsei University. All mice were housed under specific pathogen-free conditions until the start of each experiment. The animal ethics committee of the University of Yonsei approved all experimental protocols.

Preparation of P. aeruginosa strain PAO1 (PA01). PAO1 colonies were grown on a Luria-Bertani (LB) agar plate at $37^{\circ} \mathrm{C}$ overnight. The colonies were inoculated into $10 \mathrm{ml}$ fresh LB medium and grown overnight at $37^{\circ} \mathrm{C}$ with shaking at 200 r.p.m. The overnight cultures were used to inoculate fresh LB medium and grown with aeration until reaching an optical density 600 of 1 . These cultures were then used as a source of bacteria for all experiments. GFP-tagged PAO1 strains were grown at $37^{\circ} \mathrm{C}$ in $\mathrm{LB}$ with carbenicillin $\left(100 \mu \mathrm{g} \mathrm{ml}{ }^{-1}\right), \mathrm{PAO} 1 \Delta \mathrm{fliC}$ strains were grown at $37^{\circ} \mathrm{C}$ in $\mathrm{LB}$ with gentamicin $\left(20 \mu \mathrm{g} \mathrm{ml}^{-1}\right)$, and
GFP-tagged PAO1 $\Delta$ fliC strains were grown at $37^{\circ} \mathrm{C}$ in $\mathrm{LB}$ with carbenicillin $\left(100 \mu \mathrm{g} \mathrm{ml}^{-1}\right)$ and gentamicin $\left(20 \mu \mathrm{g} \mathrm{ml}^{-1}\right)$.

PAO1 infection model. Pulmonary infection of mice was performed by intranasal instillation with $4 \times 10^{5} \mathrm{CFUs}$ of PAO1 or PAO1 $\Delta$ fliC. Mock-infected mice were inoculated with phosphate-buffered saline (PBS). Mice were killed at 12 or $24 \mathrm{~h}$ after infection.

Collection of BAL, blood, and lung tissue samples. Mice were killed by administration of an overdose of tiletamine/zolazepam andxylazine. BAL fluid was obtained using $1 \mathrm{ml}$ of PBS and used for measurements of cell count, protein concentration, CFU, IgM, and cytokines. The left lung of each mouse was removed and fixed for hematoxylin-eosin staining and fibrin staining. Serum was obtained from murine blood by centrifugation (5000 r.p.m., $10 \mathrm{~min}$ ) for the measurement of cytokines and sepsis markers.

Flow cytometry. Cells obtained from BAL samples were counted and stained with Ly6G (eBiosciences, San Diego, CA), FLICA-660 (immunochemistry, Bloomington, MN), PI (Biovision, Milpitas, CA), and 7-aminoactinomycin D (7-AAD; eBiosciences). Stained cells were analyzed with a FACSverse BD flow cytometer (BD Biosciences, Sparks, MD). Isolated murine neutrophils were identified using Ly6G (eBiosciences), and isolated human neutrophils were identified using cd66b (BD Biosciences, San Jose, CA). GFP-tagged PAO1 were used in conjunction with Ly6G to quantitate neutrophil uptake of bacteria. Pyroptotic neutrophils were detected using FLICA-660 and PI or 7-AAD only. Mitochondrial ROS were measured by staining cells with $5 \mu \mathrm{M}$ MitoSOX (Invitrogen, Carlsbad, CA) for $15 \mathrm{~min}$ at $37^{\circ} \mathrm{C}$. Mitochondrial membrane potential was measured by staining cells with $100 \mathrm{~nm}$ TMRE (Abcam, Cambridge, UK) for $20 \mathrm{~min}$ at $37^{\circ} \mathrm{C}$.

Neutrophil and macrophage depletions. Neutrophils were depleted or mock-depleted by intraperitoneally administration of $0.1 \mathrm{mg}$ $\alpha$-Ly6G antibody (endotoxin-free 1A8 clone, BioXcell, West Lebanon, $\mathrm{NH}$ ) or isotype control antibody (endotoxin-free 2A3 clone, BioXcell). At $24 \mathrm{~h}$ after antibody injection, mice were challenged intranasally with $4 \times 10^{5}$ CFUs of PAO1. Alveolar macrophages were depleted by intranasal instillation of clodronate liposomes or control liposomes (50 $\mu \mathrm{l}$ per mouse) (Formumax, Suite Palo Alto, CA). Three days after the intranasal instillation of liposomes, $4 \times 10^{5} \mathrm{CFU}$ of PAO1 were instilled.

Western blotting analysis. Primary anti-CASP1 (Adipogen, San Diego, CA), anti-IL-1 $\beta$ (Biovision), and anti- $\beta$-actin (Santa Cruz, Santa Cruz, CA) (loading control) antibody were used. Western blottings were performed on cell extracts and methanol/chloroformprecipitated cell-free supernatants as previously described. ${ }^{52}$ Murine BMDNs were lysed with lysis buffer (Invitrogen), and the protein contents of the resultant extracts were quantified. Equal amounts were resolved by sodium dodecyl sulfate-polyacrylamide gel electrophoresis and transferred to nitrocellulose membranes (Millipore, Darmstadt, Germany). The membranes were incubated with primary antibodies, followed by horseradish peroxidase-conjugated secondary antibodies. Immunoreactive bands were visualized using the ECL system (Animal Genetics, Tallahassee, FL).

Preparation of mouse neutrophils. After cervical dislocation, both hind limbs were removed directly from each mouse, and a single cell suspension without erythrocytes was generated. After centrifugation (1400 r.p.m. per $5 \mathrm{~min}$ per room temperature), neutrophils were isolated via negative selection by an autoMACS column (Miltenyi Biotec, Cologne, Germany). After negative selection of neutrophils, cells were stained with anti-Ly6G antibody and analyzed by flow cytometry to determine neutrophil purity ( $>95 \%$ purity was obtained).

Isolation of human neutrophils from peripheral blood. Human neutrophils were isolated from the peripheral blood of healthy volunteers. Briefly, EDTA-treated blood was fractionated on a Ficoll-Paque Plus (GE Healthcare, Amersham, UK) gradient. 
Erythrocytes were lysed, and a $>95 \%$ pure neutrophil population was obtained.

Cytokine production. Cytokine levels in BAL samples and cultured murine BMDN supernatants were measured with a mouse IL-18 Kit (MBL, Woburn, MA), a mouse IgM Kit (eBiosciences), or a Duoset ELISA Development Kit for mouse TNF- $\alpha$, IL-6, and IL-1 $\beta$. Cytokine levels in human neutrophil supernatants were measured with a human IL-18 Kit (MBL) or a Duoset ELISA Development Kit (R\&D Systems, Minneapolis, MN) for human TNF- $\alpha$ and IL-1 $\beta$. Cytotoxicity was analyzed by measuring the release of the cytosolic enzyme LDH (Cytotox 96, Promega, Madison, WI) into the supernatants.

$\mathrm{HOCl}^{-}$detection in mouse neutrophils. Pulmonary infection of mice was performed by intranasal instillation with $4 \times 10^{5} \mathrm{CFU}$ of PAO1. Neutrophils were obtained from BAL samples taken $8 \mathrm{~h}$ after PAO1 infection. After $20 \mathrm{~m}$ incubation $\left(37^{\circ} \mathrm{C}\right)$ with $10 \mu \mathrm{M}$ HOCL probe (R19-S), cells were stained with anti-Ly6G antibody. $\mathrm{HOCl}^{-}$was detected in neutrophils by flow cytometry.

Live-cell imaging. For live-cell video microscopy, human neutrophils were attached to the confocal dish for $2 \mathrm{~h}$ and infected with GFP-tagged PAO1 or GFP-tagged PAO1 $\Delta$ fliC (multiplicity of infection (MOI) 20) and treated with the CASP1 probe FLICA-660 simultaneously. Cells were moved to a prewarmed microscope stage $\left(37^{\circ} \mathrm{C} / 5 \% \mathrm{CO}_{2}\right)$, and the images were acquired every $20 \mathrm{~s}$ on a confocal microscope (Carl Zeiss, Oberkochen, Germany; LSM 780). The movie plays at a rate of two frames per second.

Quantification of fibrin deposition. Formalin-fixed mouse left lung lobes were dehydrated gradually in ethanol, embedded in paraffin, and cut into $5-\mu \mathrm{m}$ sections. Heat-induced epitope retrieval was then performed before staining. The tissue sections were permeabilized with $0.2 \%$ Triton X-100 for 10 min and blocked with PBS containing $1 \%$ bovine serum albumin and $10 \%$ normal goat serum. Sections were then immunostained with anti-fibrin antibody (Dako, Glostrub, Denmark). After extensive washing with PBS, tissue sections were incubated with Alexa488-conjugated anti-rabbit IgG antibody. After washing with PBS, tissue sections were stained with DAPI (4,6-diamidino-2-phenylindole) and fibrin, and DAPI colocalization was analyzed on a confocal microscope (Carl Zeiss; LSM 700).

Transmission electron microscopy. Neutrophils were fixed with glutaraldehyde as previously described. ${ }^{53}$

BMDM culture and treatment. BMDMs were cultured in Dulbecco's modified Eagle's medium supplemented with $10 \%$ fetal bovine serum, $1 \%$ penicillin/streptomycin, and 25\% L929 mouse fibroblast supernatant. PAO1 were added to cultured BMDMs at an MOI of 5 followed by incubation at $37^{\circ} \mathrm{C}$ for $2 \mathrm{~h}$.

Inhibitor study. Mice were pretreated intraperitoneally with the CASP1 inhibitor Ac-YVAD-CHO $(30 \mu \mathrm{g}$ per mouse; Cayman Chemical, Ann Arbor, MI) $1 \mathrm{~h}$ prior to infection. Control mice were treated with dimethyl sulfoxide for CASP1 inhibitor. Isolated murine neutrophils were pretreated with dimethyl sulfoxide or the CASP1 inhibitor AC-YVAD-CHO $\left(50 \mu \mathrm{g} \mathrm{ml}^{-1}\right) 30 \mathrm{~min}$ prior to infection with GFP-tagged PAO1 (PAO1-GFP).

In vitro neutrophil killing assay. PAO1 were added to freshly isolated neutrophils at an MOI of 5 and 20 followed by incubation at $37^{\circ} \mathrm{C}$ for $30 \mathrm{~min}$. Subsequently, neutrophils were incubated with gentamicin $\left(20 \mu \mathrm{g} \mathrm{ml}^{-1}\right.$; Gibco, Grand Island, NY) for $30 \mathrm{~min}$ at $37^{\circ} \mathrm{C}$ to kill bacteria. After $30 \mathrm{~min}$, neutrophils were lysed with $0.1 \%$ Triton X-100. Lysates were plated onto LB agar and incubated overnight at $37^{\circ} \mathrm{C}$. The next day, the colonies were counted and relative phagocytosis was determined by CFU counts.

Time-lapse recording of human neutrophil pyroptosis (video clip). Live-cell video microscopic experiment shows phagocytosis of GFPtagged PAO1 (green) and GFP-tagged PAO1 $\Delta$ fliC (green) by human neutrophils. Human neutrophils were infected with GFP-tagged PAO1 or GFP-tagged PAO1 $\Delta$ fliC (MOI 20) after $2 \mathrm{~h}$ of attachment to the confocal dish. Cells were moved to a prewarmed microscope stage $\left(37^{\circ} \mathrm{C} / 5 \% \mathrm{CO}_{2}\right) 5$ min after infection with GFP-tagged PAO1 or GFPtagged PAO1 $\Delta$ fliC. Images were acquired every $20 \mathrm{~s}$. The movie plays at a rate of two frames per second.

Statistical analysis. Comparisons of two samples were made by an unpaired Student's $t$-test, whereas analysis of variance followed by Tukey's post-hoc test was performed to compare multiple samples.

The Kaplan-Meier log-rank test was used for the statistical analysis of survival experiments.

$P$-values $<0.05$ were considered statistically significant. In each figure legend, s.e.m. stands for the between-subjects s.e.m. All statistical analyses were performed using the SPSS software (IBM, Armonk, NY).

Study approval. All animal experiments and protocols were approved by the Yonsei University College of Medicine Institutional Animal Care and Use Committee. Human blood samples from healthy volunteers were obtained at Severance Hospital (Seoul, Korea). This study was approved by the Institutional Review Board of Severance Hospital, Yonsei University College of Medicine (4-2015-0007). Informed consent was obtained directly from each subject and documented in writing before the start of any study-related procedure.

SUPPLEMENTARY MATERIAL is linked to the online version of the paper at http://www.nature.com/mi

\section{ACKNOWLEDGMENTS}

This research was supported by the Basic Science Research Program through the NRF, which is funded by the Ministry of Education (2013R1A1A2008511). This work was also supported by an NRF grant funded by the Ministry of Science, ICT \& Future Planning (2012M3A9C5048709 and 2013M3A9D5072550). We thank Yonsei-Carl Zeiss Advanced Imaging Center, Yonsei University College of Medicine for technical assistance. We also thank D.S. Jang for his excellent support with medical illustration.

\section{AUTHOR CONTRIBUTIONS}

J.C.R. designed and performed experiments, analyzed data, generated figures, and wrote the manuscript; M.J.K., Y.K., and J.H.O. assisted with knockout mouse breeding and performed mouse infection experiments; S.S.Y and S.J.S helped conceive the study of innate immunity against $P$. aeruginosa infections; J.H.Y. helped with data interpretation; and J.H.R. supervised the project, designed experiments, helped with data interpretation, participated in data analysis, and wrote the manuscript.

\section{DISCLOSURE}

The authors declared no conflict of interest.

c) 2017 Society for Mucosal Immunology

\section{REFERENCES}

1. Britigan, B.E., Rasmussen, G.T. \& Cox, C.D. Augmentation of oxidant injury to human pulmonary epithelial cells by the Pseudomonas aeruginosa siderophore pyochelin. Infect. Immun. 65, 1071-1076 (1997).

2. Hauser, A.R. Ventilator-associated pneumonia caused by Pseudomonas aeruginosa: cap your needles!. Crit. Care Med. 40, 2503-2504 (2012).

3. Kurahashi, K. et al. Pathogenesis of septic shock in Pseudomonas aeruginosa pneumonia. J. Clin. Invest. 104, 743-750 (1999).

4. Koh, A.Y., Priebe, G.P., Ray, C., Van Rooijen, N. \& Pier, G.B. Inescapable need for neutrophils as mediators of cellular innate immunity to acute Pseudomonas aeruginosa pneumonia. Infect. Immun. 77, 5300-5310 (2009).

5. Lavoie, E.G., Wangdi, T. \& Kazmierczak, B.I. Innate immune responses to Pseudomonas aeruginosa infection. Microbes Infect. 13, 1133-1145 (2011). 
6. Miao, E.A. et al. Innate immune detection of the type III secretion apparatus through the NLRC4 inflammasome. Proc. Natl. Acad. Sci. USA 107, 3076-3080 (2010).

7. Miao, E.A. et al. Cytoplasmic flagellin activates caspase- 1 and secretion of interleukin 1 beta via Ipaf. Nat. Immunol. 7, 569-575 (2006).

8. Franchi, L. et al. Cytosolic flagellin requires Ipaf for activation of caspase-1 and interleukin 1 beta in salmonella-infected macrophages. Nat. Immunol. 7, 576-582 (2006).

9. Zhao, Y. et al. The NLRC4 inflammasome receptors for bacterial flagellin and type III secretion apparatus. Nature 477, 596-600 (2011).

10. Halff, E.F., Diebolder, C.A., Versteeg, M., Schouten, A., Brondijk, T.H. \& Huizinga, E.G. Formation and structure of a NAIP5-NLRC4 inflammasome induced by direct interactions with conserved $\mathrm{N}$ - and $\mathrm{C}$-terminal regions of flagellin. J. Biol. Chem. 287, 38460-38472 (2012).

11. Broz, P., Newton, K., Lamkanfi, M., Mariathasan, S., Dixit, V.M. \& Monack, D.M. Redundant roles for inflammasome receptors NLRP3 and NLRC4 in host defense against Salmonella. J. Exp. Med. 207, 1745-1755 (2010).

12. Pereira, M.S., Morgantetti, G.F., Massis, L.M., Horta, C.V., Hori, J.I. \& Zamboni, D.S. Activation of NLRC4 by flagellated bacteria triggers caspase-1-dependent and -independent responses to restrict Legionella pneumophila replication in macrophages and in vivo. J. Immunol. 187, 6447-6455 (2011).

13. Chen, K.W. \& Schroder, K. Antimicrobial functions of inflammasomes. Curr. Opin. Microbiol. 16, 311-318 (2013).

14. Franchi, L., Munoz-Planillo, R. \& Nunez, G. Sensing and reacting to microbes through the inflammasomes. Nat. Immunol. 13, 325-332 (2012).

15. Netea, M.G., Simon, A., van de Veerdonk, F., Kullberg, B.J., Van der Meer, J.W. \& Joosten, L.A. IL-1beta processing in host defense: beyond the inflammasomes. PLoS Pathog. 6, e1000661 (2010).

16. Miao, E.A. et al. Caspase-1-induced pyroptosis is an innate immune effector mechanismagainst intracellular bacteria. Nat. Immunol. 11, 1136-1142 (2010).

17. Bergsbaken, T., Fink, S.L. \& Cookson, B.T. Pyroptosis: host cell death and inflammation. Nat. Rev. Microbiol. 7, 99-109 (2009).

18. Labbe, K. \& Saleh, M. Cell death in the host response to infection. Cell Death Differ. 15, 1339-1349 (2008).

19. Lamkanfi, M. \& Dixit, V.M. Manipulation of host cell death pathways during microbial infections. Cell Host Microbe 8, 44-54 (2010).

20. Faure, E. et al. Pseudomonas aeruginosa type-3 secretion system dampens host defense by exploiting the NLRC4-coupled inflammasome. Am. J. Respir. Crit. Care Med. 189, 799-811 (2014).

21. Cohen, T.S. \& Prince, A.S. Activation of inflammasome signaling mediates pathology of acute $P$. aeruginosa pneumonia. J. Clin. Invest. 123, 16301637 (2013).

22. Lekstrom-Himes, J.A. \& Gallin, J.I. Immunodeficiency diseases caused by defects in phagocytes. N. Engl. J. Med. 343, 1703-1714 (2000).

23. Chen, K.W. et al. The neutrophil NLRC4 inflammasome selectively promotes IL-1 beta maturation without pyroptosis during acute Salmonella challenge. Cell Rep. 8, 570-582 (2014).

24. Lightfield, K.L. et al. Differential requirements for NAIP5 in activation of the NLRC4 inflammasome. Infect. Immun. 79, 1606-1614 (2011).

25. Patankar, Y.R., Mabaera, R. \& Berwin, B. Differential ASC requirements reveal a key role for neutrophils and a noncanonical IL-1beta response to Pseudomonas aeruginosa. Am. J. Physiol. Lung Cell Mol. Physiol. 309, L902-L913 (2015).

26. Pollock, J.D. et al. Mouse model of X-linked chronic granulomatous disease, an inherited defect in phagocyte superoxide production. Nat. Genet. 9, 202-209 (1995).

27. Rosen, H. \& Klebanoff, S.J. Bactericidal activity of a superoxide aniongenerating system. A model for the polymorphonuclear leukocyte. J. Exp. Med. 149, 27-39 (1979).

28. Hampton, M.B., Kettle, A.J. \& Winterbourn, C.C. Inside the neutrophil phagosome: oxidants, myeloperoxidase, and bacterial killing. Blood 92, 3007-3017 (1998).

29. Silveira, T.N. \& Zamboni, D.S. Pore formation triggered by Legionella spp. is an Nlrc4 inflammasome-dependent host cell response that precedes pyroptosis. Infect. Immun. 78, 1403-1413 (2010).
30. Suzuki, T. et al. Differential regulation of caspase-1 activation, pyroptosis, and autophagy via Ipaf and ASC in Shigella-infected macrophages. PLOS Pathog. 3, e111 (2007).

31. Verma, A., Arora, S.K., Kuravi, S.K. \& Ramphal, R. Roles of specific amino acids in the $\mathrm{N}$ terminus of $P$ seudomonas aeruginosa flagellin and of flagellin glycosylation in the innate immune response. Infect. Immun. 73, 82378246 (2005).

32. Feuillet, V. et al. Involvement of Toll-like receptor 5 in the recognition of flagellated bacteria. Proc. Natl. Acad. Sci. USA 103, 12487-12492 (2006).

33. Geddes, K., Cruz, F. \& Heffron, F. Analysis of cells targeted by Salmonella type III secretion in vivo. PLoS Pathog. 3, e196 (2007).

34. Rogers, D.E. \& Tompsett, R. The survival of staphylococci within human leukocytes. J. Exp. Med. 95, 209-230 (1952).

35. Casey, S.G., Shafer, W.M. \& Spitznagel, J.K. Neisseria gonorrhoeae survive intraleukocytic oxygen-independent antimicrobial capacities of anaerobic and aerobic granulocytes in the presence of pyocin lethal for extracellular gonococci. Infect. Immun. 52, 384-389 (1986).

36. van Zandbergen, G. et al. Chlamydia pneumoniae multiply in neutrophil granulocytes and delay their spontaneous apoptosis. J. Immunol. 172, 1768-1776 (2004).

37. Chen, S.M., Dumler, J.S., Bakken, J.S. \& Walker, D.H. Identification of a granulocytotropic Ehrlichia species as the etiologic agent of human disease. J. Clin. Microbiol. 32, 589-595 (1994).

38. Arlehamn, C.S. \& Evans, T.J. Pseudomonas aeruginosa pilin activates the inflammasome. Cell Microbiol. 13, 388-401 (2011).

39. Franchi, L., Stoolman, J., Kanneganti, T.D., Verma, A., Ramphal, R. \& Nunez, G. Critical role for Ipaf in Pseudomonas aeruginosa-induced caspase-1 activation. Eur. J. Immunol. 37, 3030-3039 (2007).

40. Miao, E.A., Ernst, R.K., Dors, M., Mao, D.P. \& Aderem, A. Pseudomonas aeruginosa activates caspase 1 through Ipaf. Proc. Natl. Acad. Sci. USA 105, 2562-2567 (2008).

41. Sutterwala, F.S., Mijares, L.A., Li, L., Ogura, Y., Kazmierczak, B.I. \& Flavell, R.A. Immune recognition of Pseudomonas aeruginosa mediated by the IPAF/NLRC4 inflammasome. J. Exp. Med. 204, 3235-3245 (2007).

42. Bakele, M. et al. Localization and functionality of the inflammasome in neutrophils. J. Biol. Chem. 289, 5320-5329 (2014).

43. Karmakar, M., Sun, Y., Hise, A.G., Rietsch, A. \& Pearlman, E. Cutting edge: IL-1beta processing during Pseudomonas aeruginosa infection is mediated by neutrophil serine proteases and is independent of NLRC4 and caspase-1. J. Immunol. 189, 4231-4235 (2012).

44. Mankan, A.K., Dau, T., Jenne, D. \& Hornung, V. The NLRP3/ASC/ Caspase- 1 axis regulates $\mathrm{IL}-1$ beta processing in neutrophils. Eur. J. Immunol. 42, 710-715 (2012).

45. Pereira, M.S., Marques, G.G., Dellama, J.E. \& Zamboni, D.S. The NIrc4 inflammasome contributes to restriction of pulmonary infection by flagellated Legionella spp. that trigger pyroptosis. Front. Microbiol. 2, 33 (2011).

46. Cho, J.S. et al. Neutrophil-derived IL-1beta is sufficient for abscess formation in immunity against Staphylococcus aureus in mice. PLOS Pathog. 8, e1003047 (2012).

47. Kayagaki, N. et al. Non-canonical inflammasome activation targets caspase-11. Nature 479, 117-121 (2011).

48. Brodsky, I.E. \& Medzhitov, R. Pyroptosis: macrophage suicide exposes hidden invaders. Curr. Biol. 21, R72-R75 (2011).

49. Rimessi, A., Bezzerri, V., Patergnani, S., Marchi, S., Cabrini, G. \& Pinton, P. Mitochondrial $\mathrm{Ca} 2+$-dependent NLRP3 activation exacerbates the Pseudomonas aeruginosa-driven inflammatory response in cystic fibrosis. Nat. Commun. 6, 6201 (2015).

50. Jabir, M.S. et al. Mitochondrial damage contributes to Pseudomonas aeruginosa activation of the inflammasome and is downregulated by autophagy. Autophagy 11, 166-182 (2015).

51. Koller, B. et al. TLR expression on neutrophils at the pulmonary site of infection: TLR1/TLR2-mediated up-regulation of TLR5 expression in cystic fibrosis lung disease. J. Immunol. 181, 2753-2763 (2008).

52. Gross, O. Measuring the inflammasome. Methods Mol. Biol. 844, 199-222 (2012).

53. Chen, Z.H. et al. Egr-1 regulates autophagy in cigarette smoke-induced chronic obstructive pulmonary disease. PLoS One 3, e3316 (2008). 